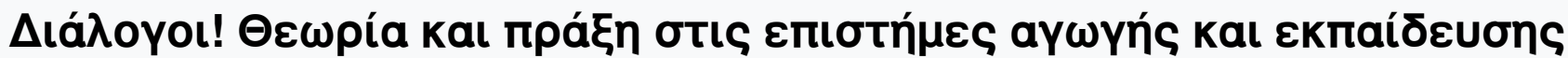

Tóp. 2 (2016)

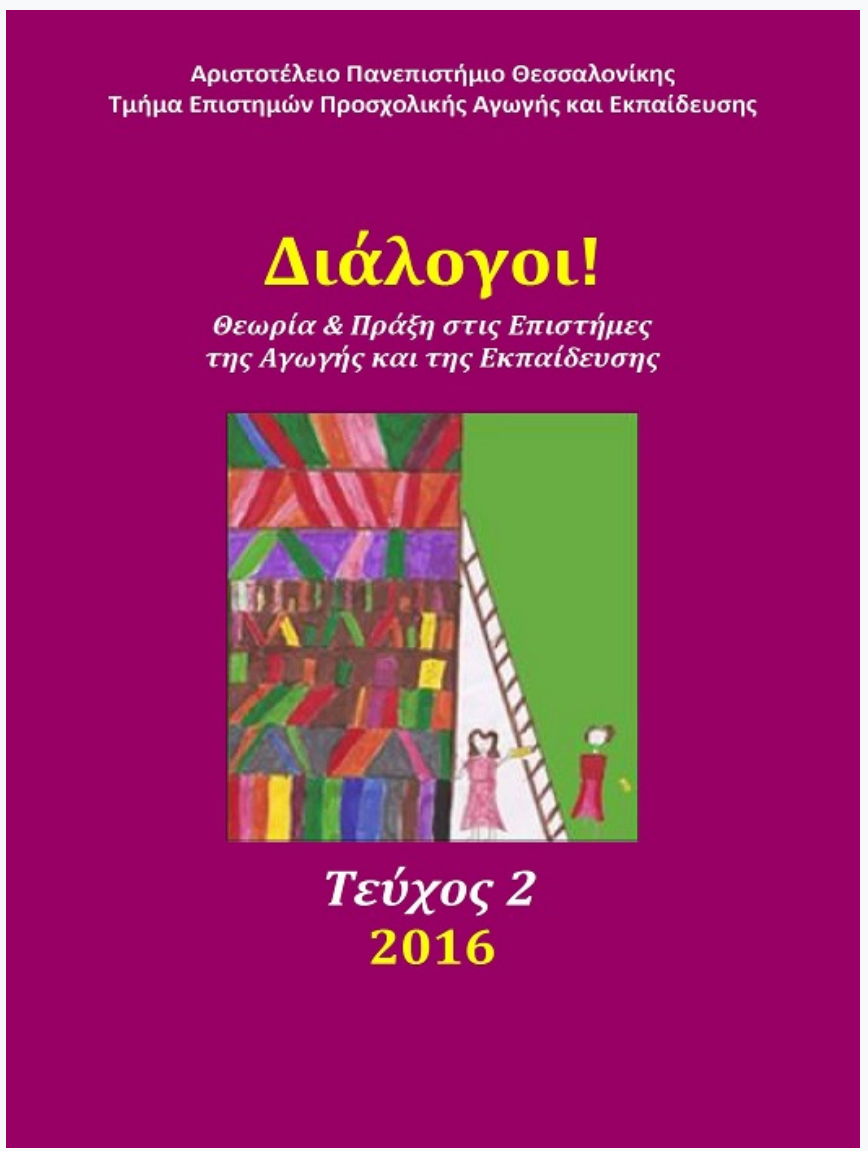

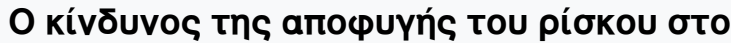 naıxvíoı}

\section{Melanthia Kontopoulou}

doi: $10.12681 /$ dial.10512

Copyright (C) 2016, Melanthia Kontopoulou

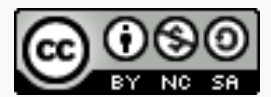

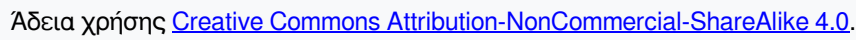

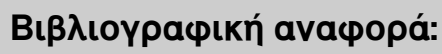

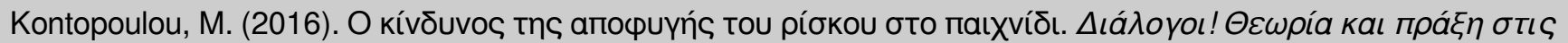

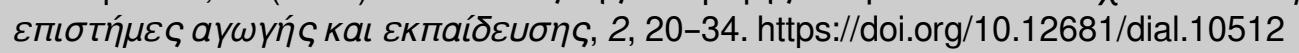




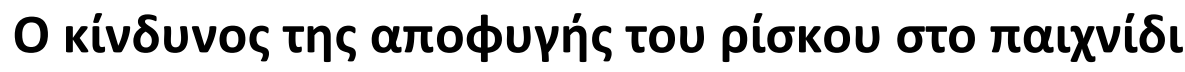

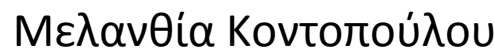

АПӨ

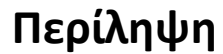

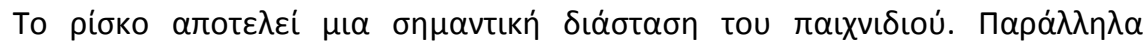

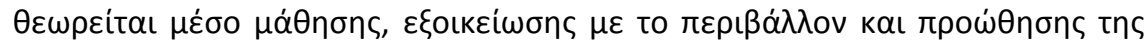

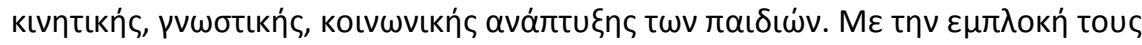

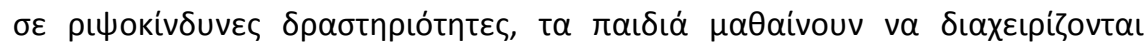

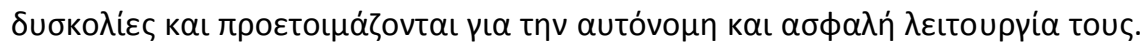

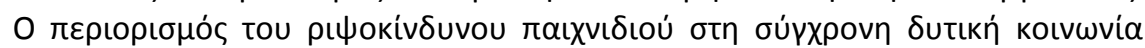

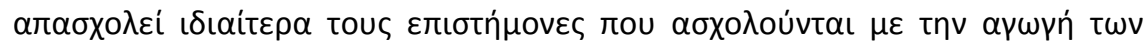

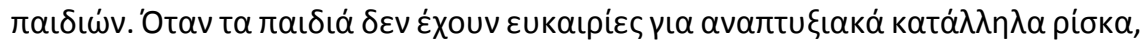

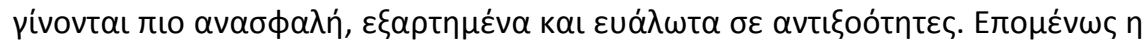

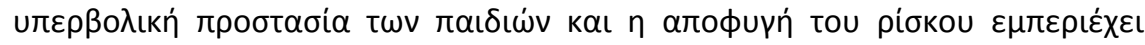

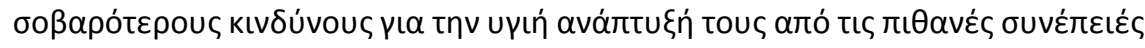

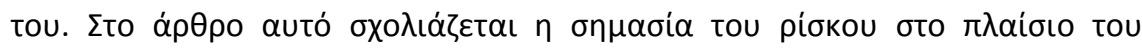

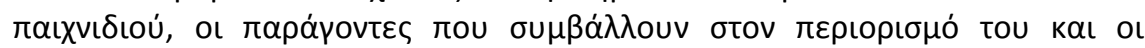

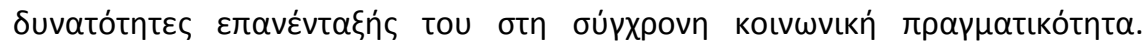

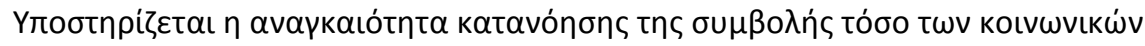

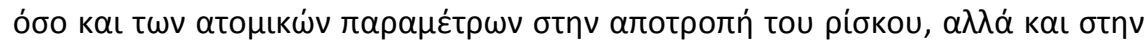

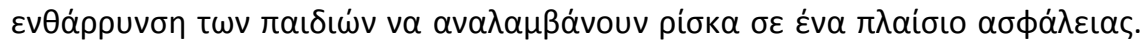

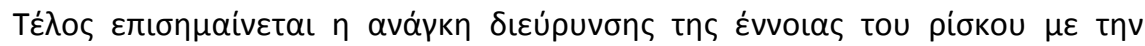

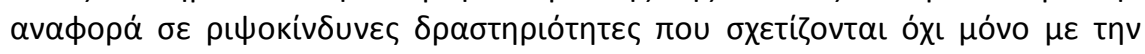

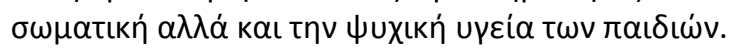

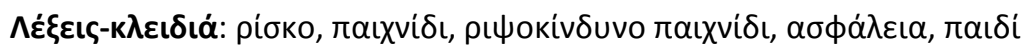

\begin{abstract}
Risk is an important and highly appealing dimension of play. At the same time, it is considered as a means to learn, familiarize oneself with the environment and promote the motor, cognitive and social development of children. With their gradual involvement in risky activities, in the context of play, children learn to deal with difficulties, to become autonomous and function safely.

Scholars and researchers ascertain a restriction of risky play in contemporary western societies, due to an overprotective attitude towards children. The reasons are associated with environmental and institutional parameters, the views about childhood and the role of adults. In addition, the uncertainty experienced by adults themselves and their difficulty to become familiar with the conditions of contemporary reality and the new dangers, discourages risk acceptance, even in the context of play. However risk avoidance entails more risks for healthy development than its possible consequences. When children do
\end{abstract}

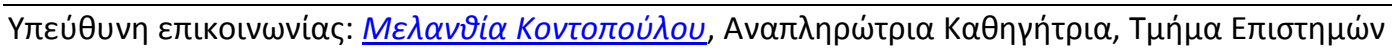

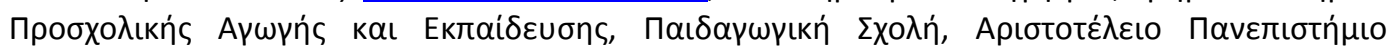

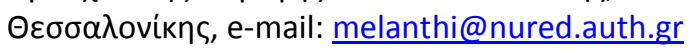


not have opportunities for developmentally appropriate risks and they do not learn how to manage them, they become insecure and vulnerable to adversities. It is necessary therefore to explore ways of reintegrating the risk in play.

The balance between protection and encouragement of risk taking is a fundamental requirement for children's education. To offer the risky activities without exposing children to serious dangers, appropriate material conditions are necessary. However adults' attitudes towards risky play are more important than the material environment. Basic premise is their own resilience and ability to support children in acquiring the necessary skills for the management of risk. The role of society and educational policy is also important. The State must have a supportive attitude towards adults who have the responsibility of children. In addition, the educational context could become a space where children's disposition and ability to take risks are encouraged. Teachers' responsibility should not be focused exclusively on child protection but mainly on shaping capable and resilient people who can handle danger in all its forms.

Although there are many scientific references on risky play, many issues are still open to investigation. There is a need to clarify the term "developmentally appropriate risk" as well as to examine the possibilities of integrating risky play in the respective cultural reality. In addition, while risk is associated mainly with physical safety and health, it could be extended to risky conditions concerning overall healthy development and psychosocial wellbeing.

Keywords: risk, play, risky play, child

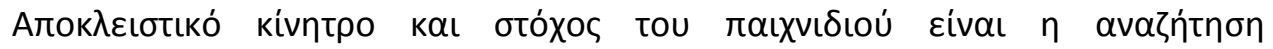

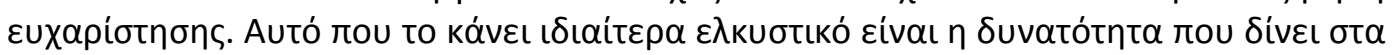

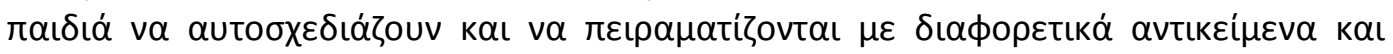

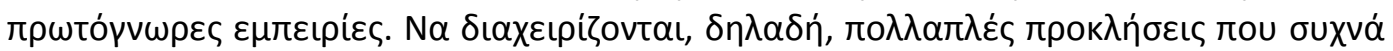

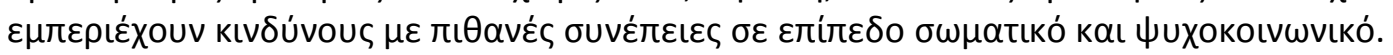

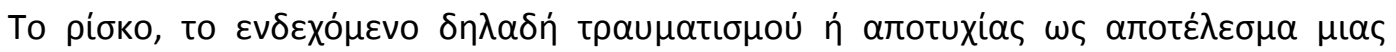

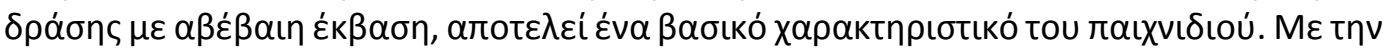

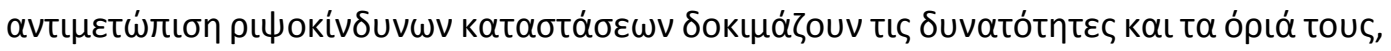

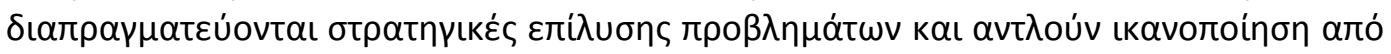

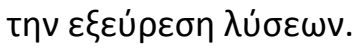

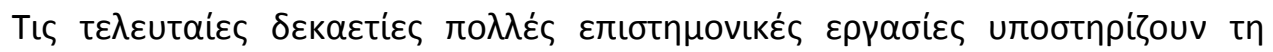

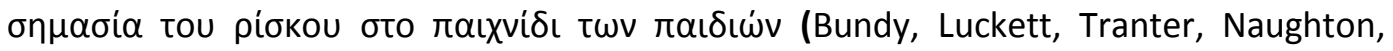
Wyver, Ragen, \& Spies, 2009. Sandseter, 2010, 2012 · Sandseter \& Kennair, 2011. Smith, 1998. Stephenson, 2003. Wyver, Tranter, Naughton, Little, Sandseter, \& Bundy, 2010).

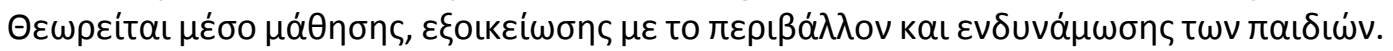

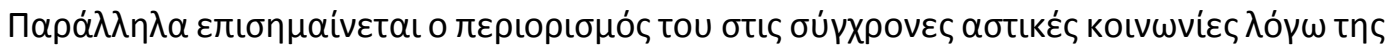

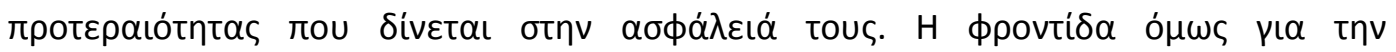

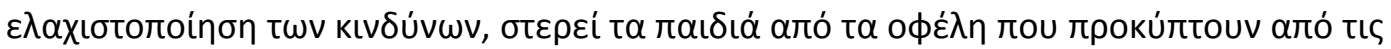

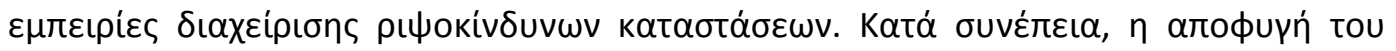

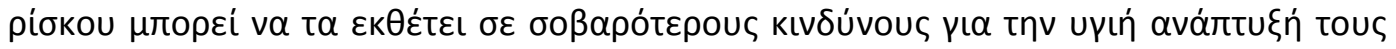
a

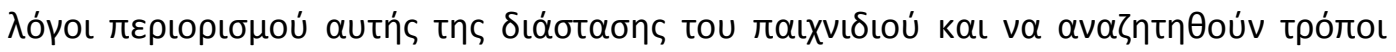

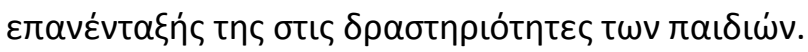




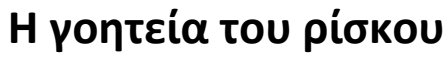

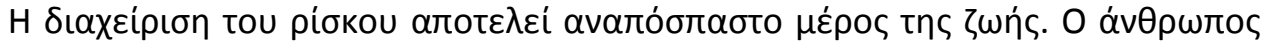

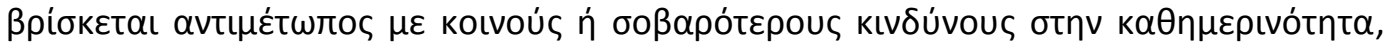

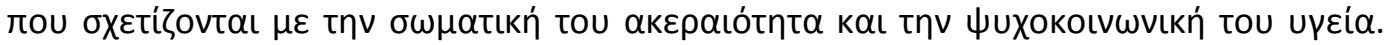

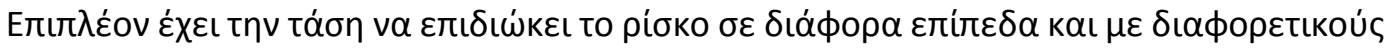

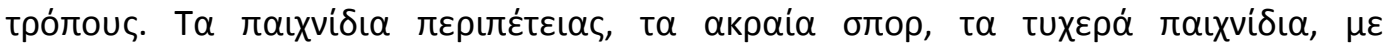

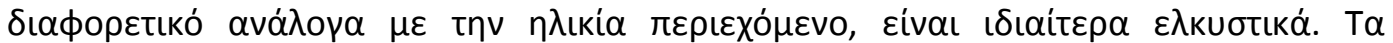

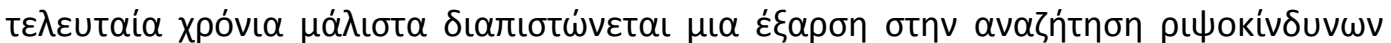

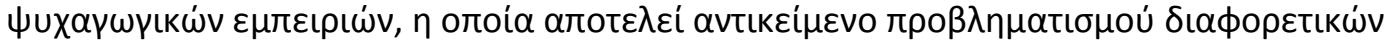

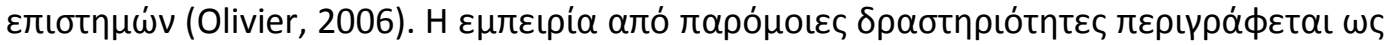

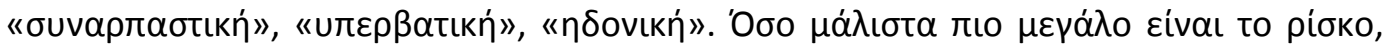

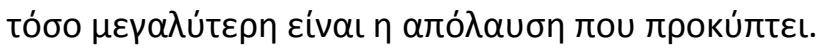

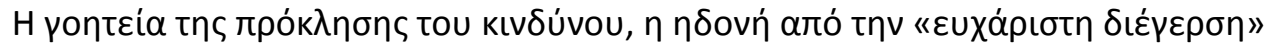

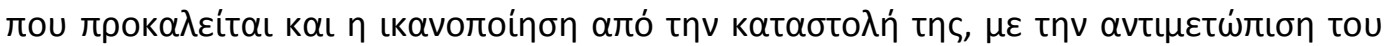

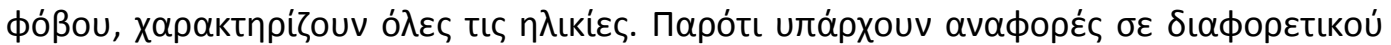

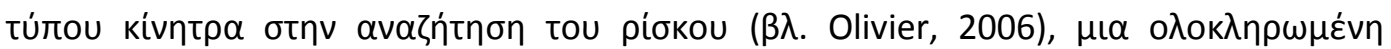

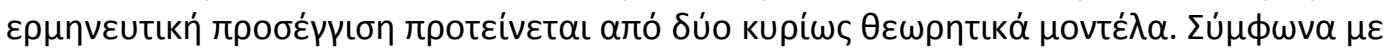

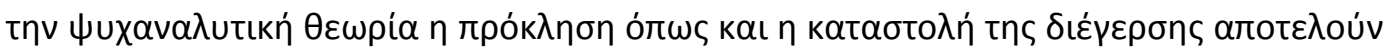

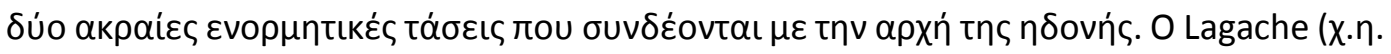

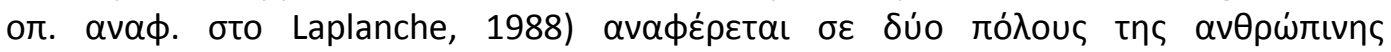

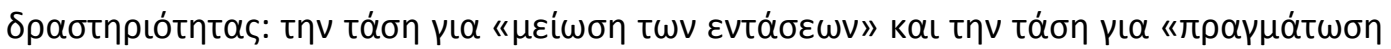

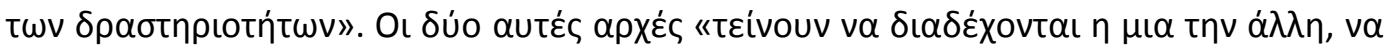

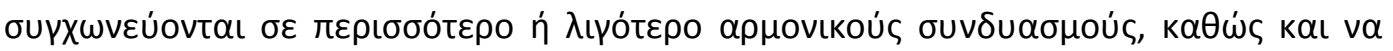

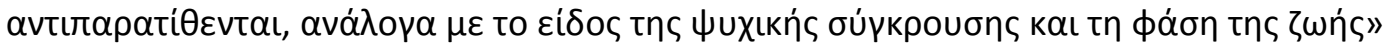

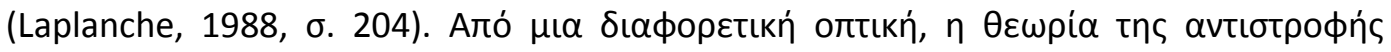

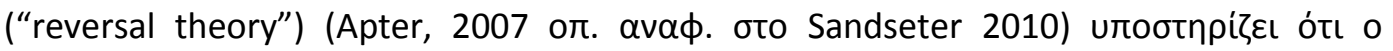

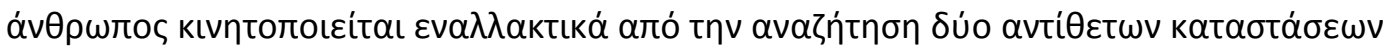

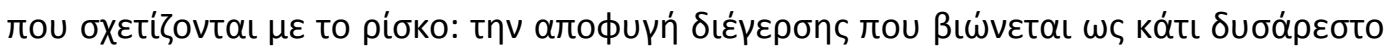

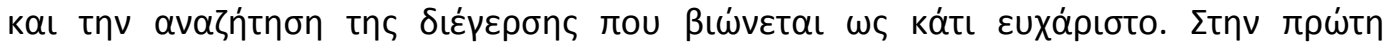

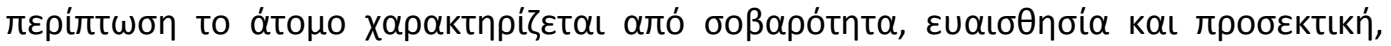

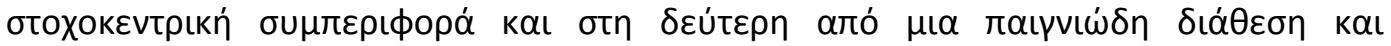

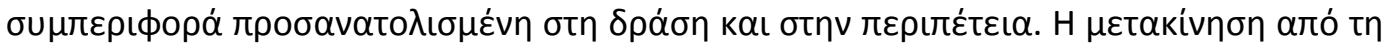

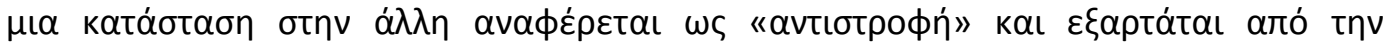

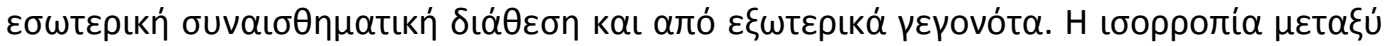

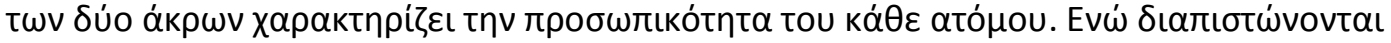

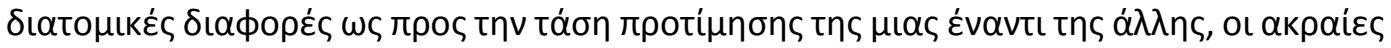

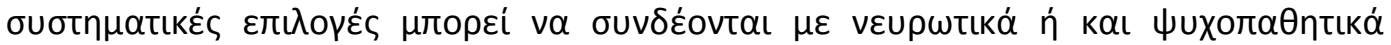

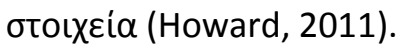

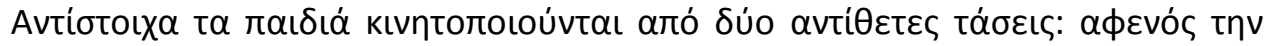

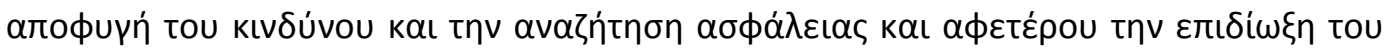

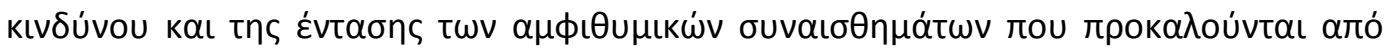

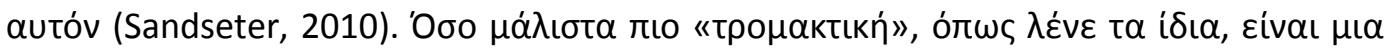

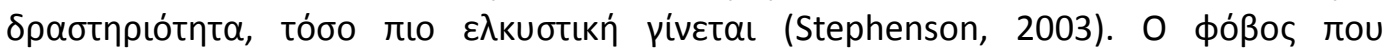

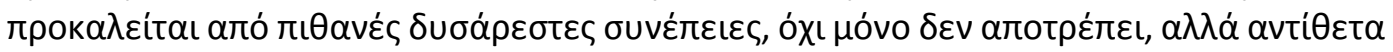

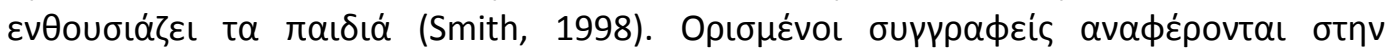

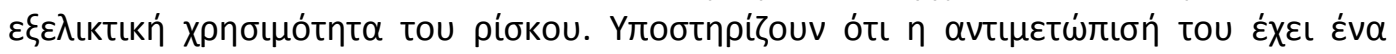




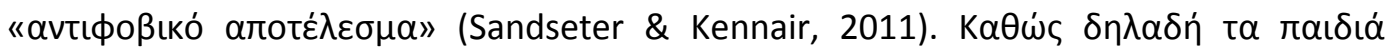

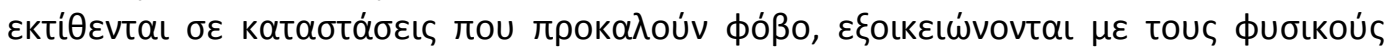

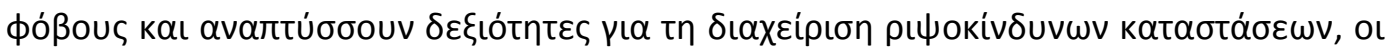

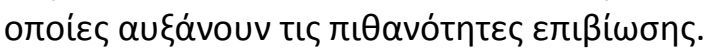

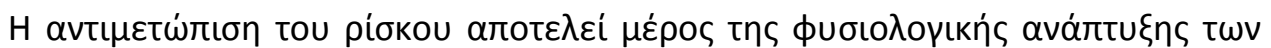

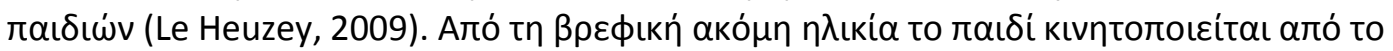

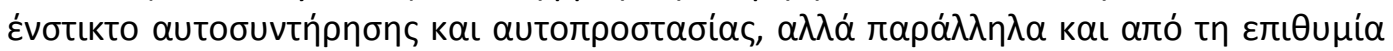

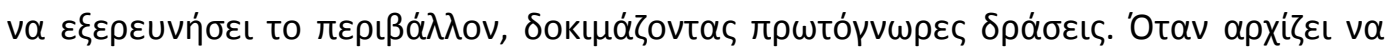

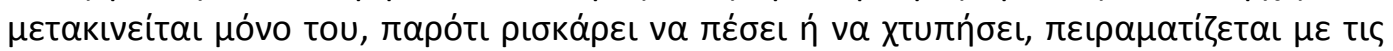

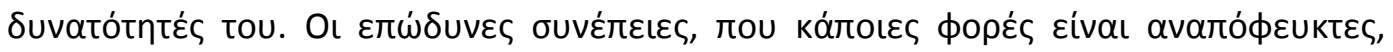

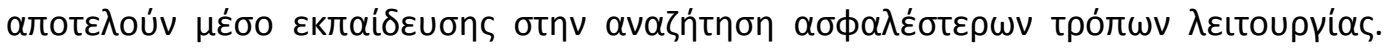

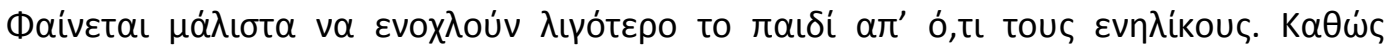

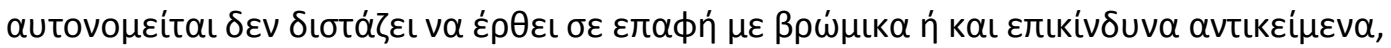

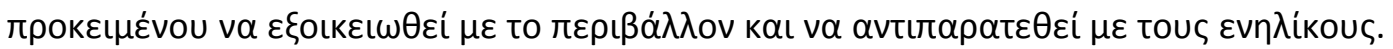

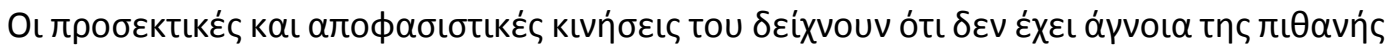

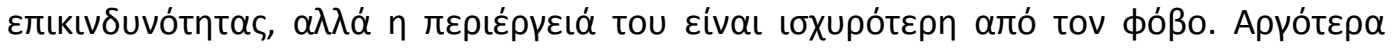

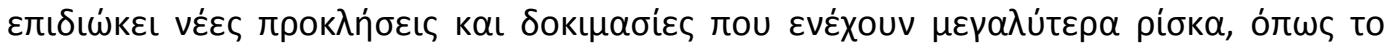

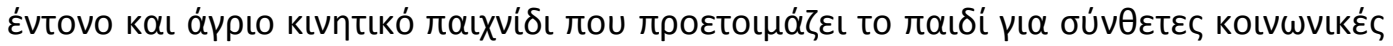

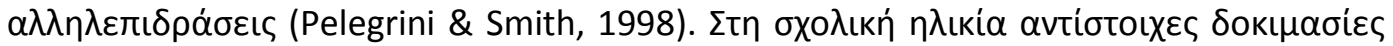

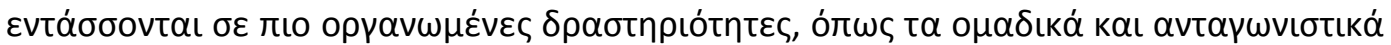

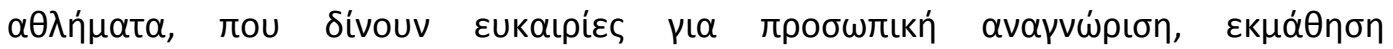

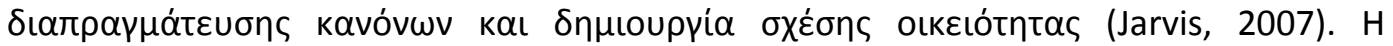

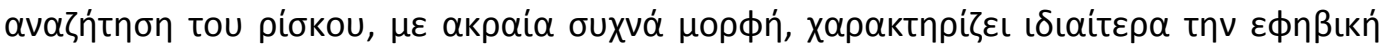

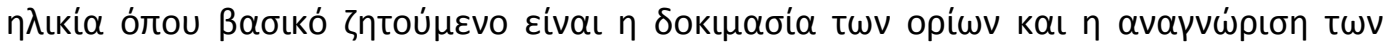

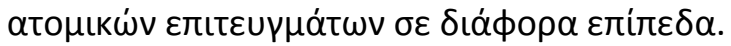

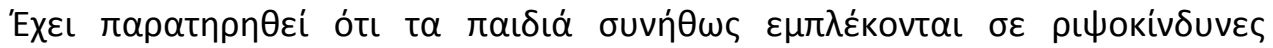

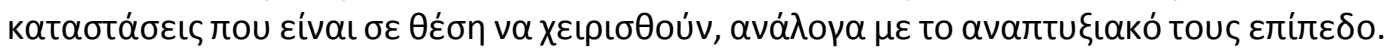

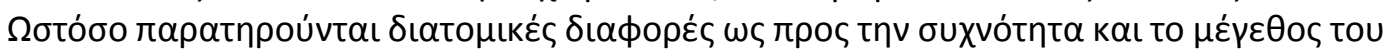

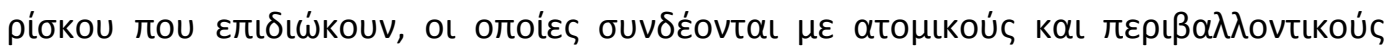

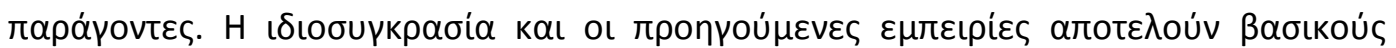

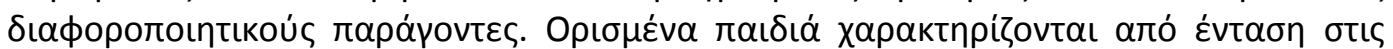

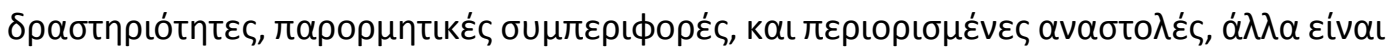

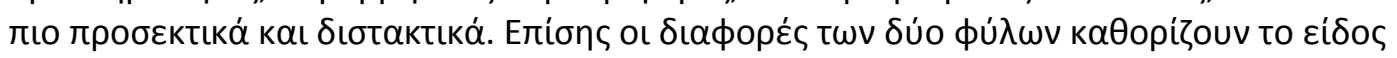

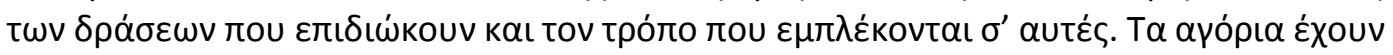

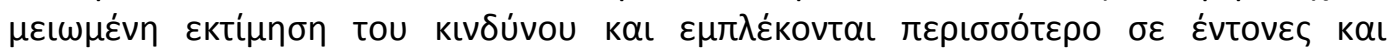

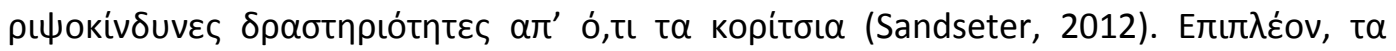

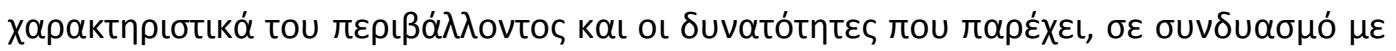

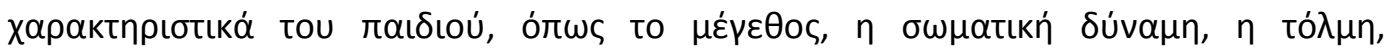

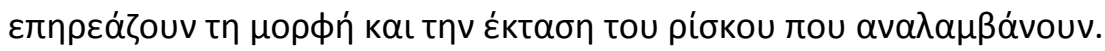

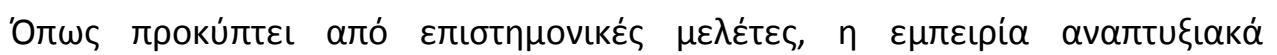

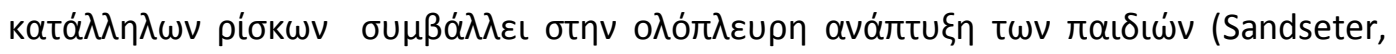

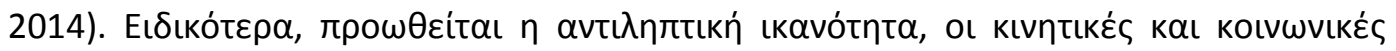

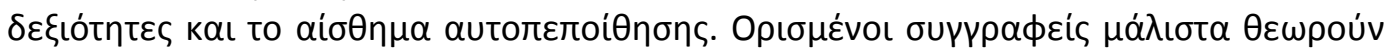

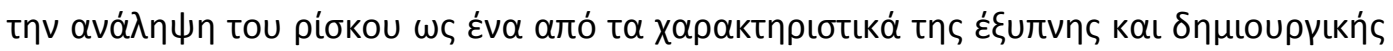

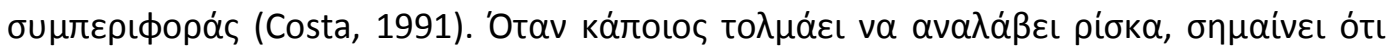
a 


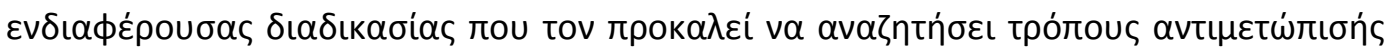

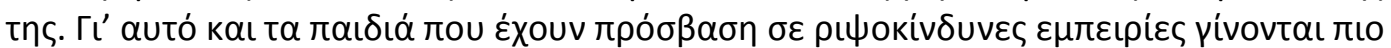

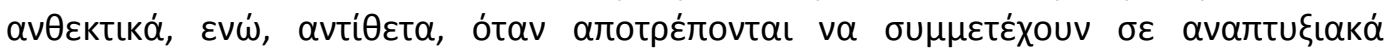

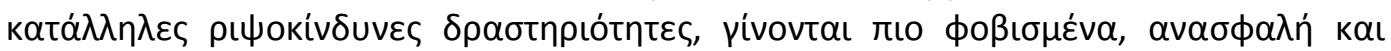
$\varepsilon \xi \alpha \rho \tau \eta \mu \varepsilon ́ v \alpha$.

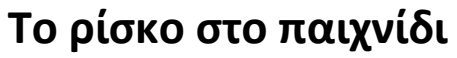

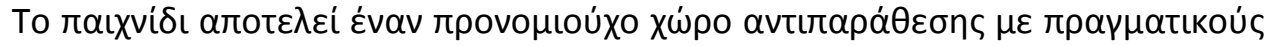

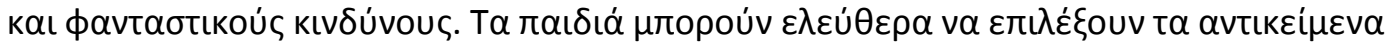

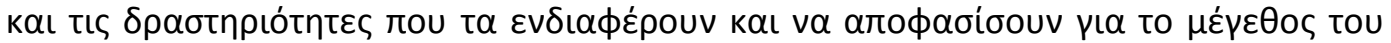

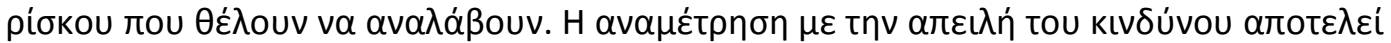

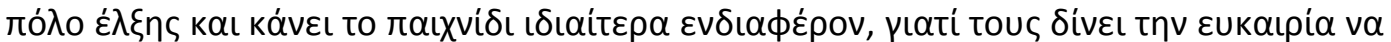

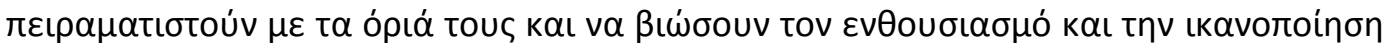

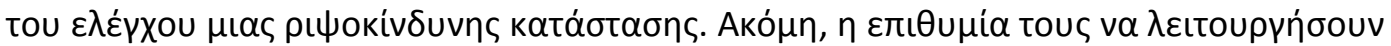

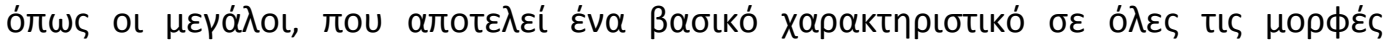

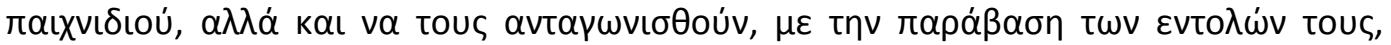

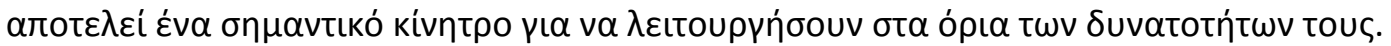

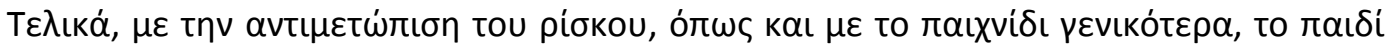

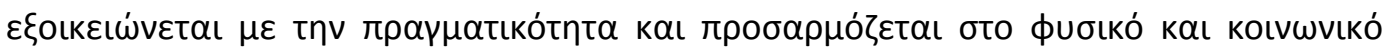
$\pi \varepsilon \rho \iota \beta \dot{\alpha} \lambda \lambda$ ov.

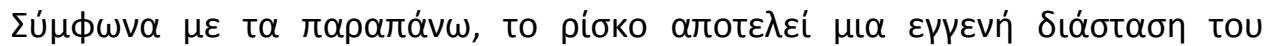

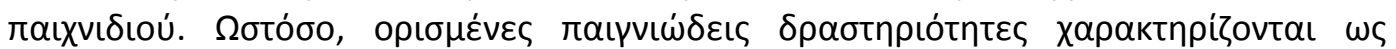

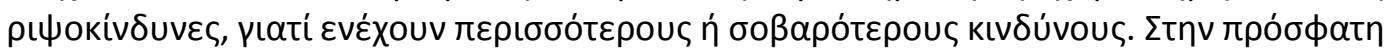

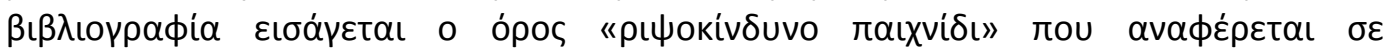

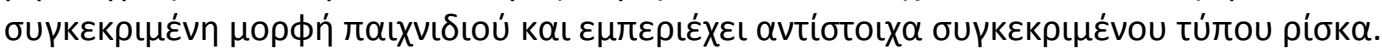

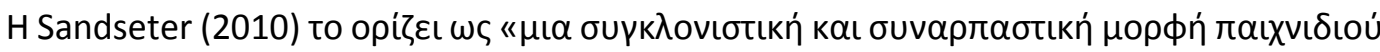

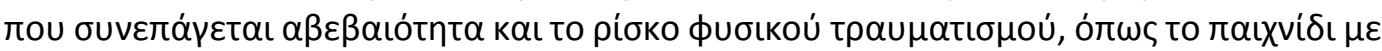

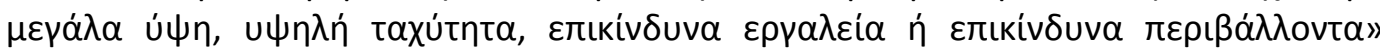

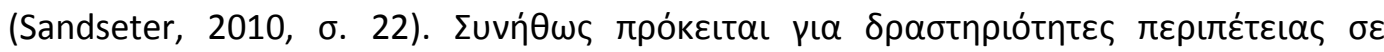

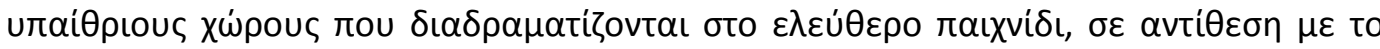

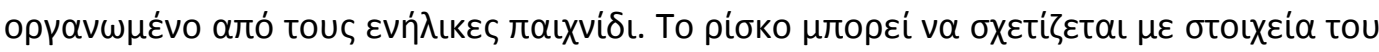

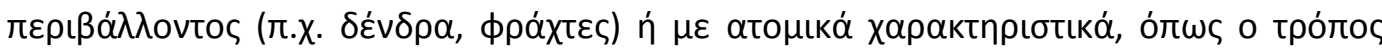

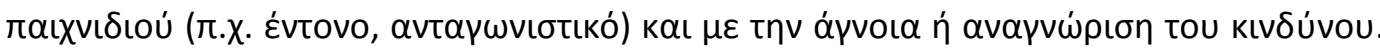

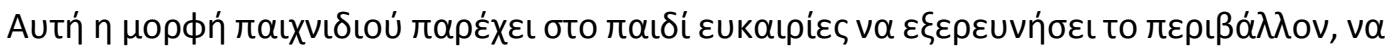

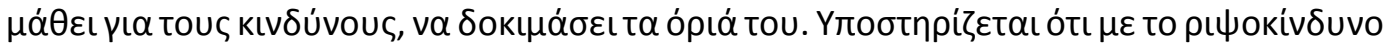

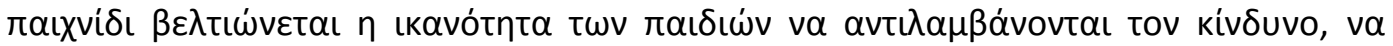

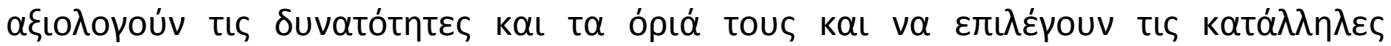

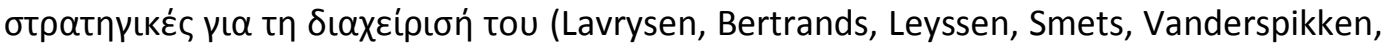

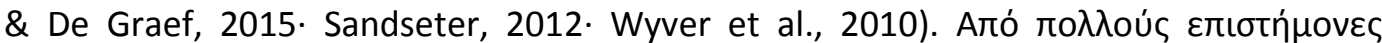

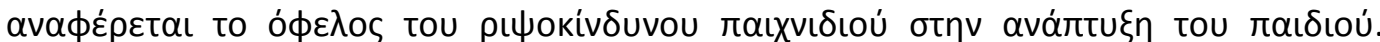

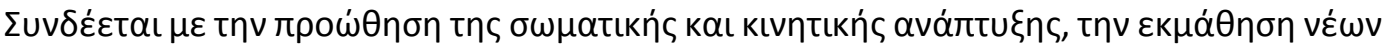

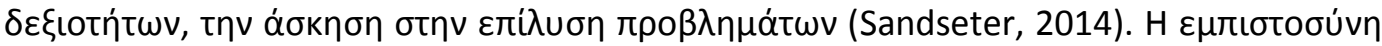

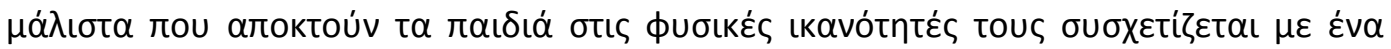

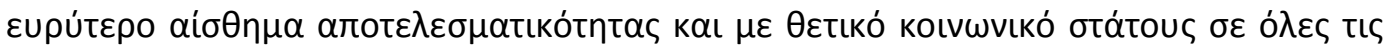

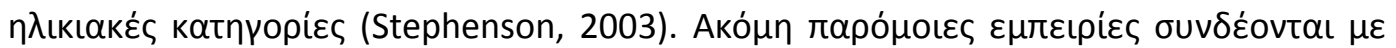




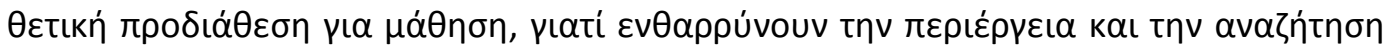

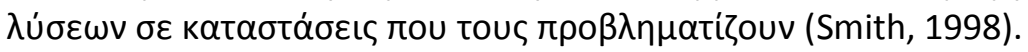

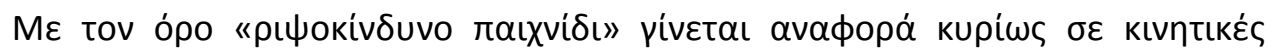

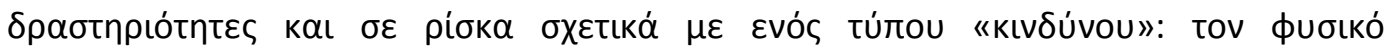

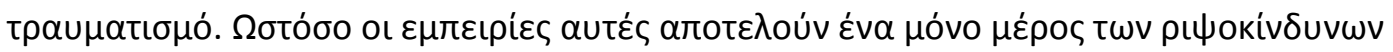

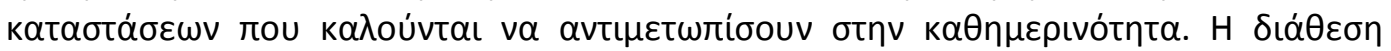

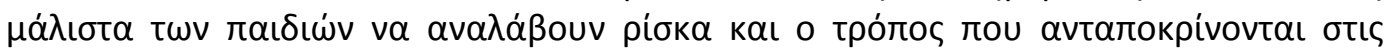

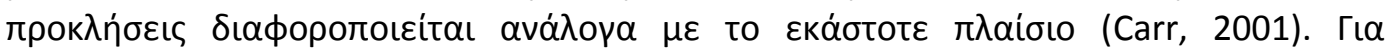

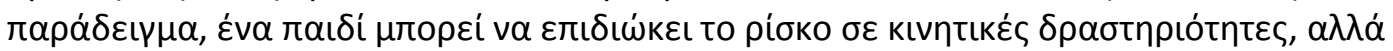

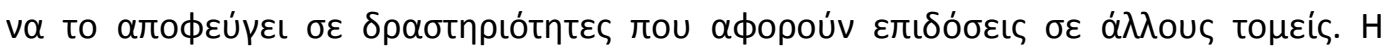

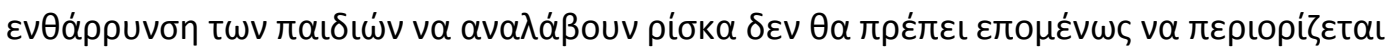

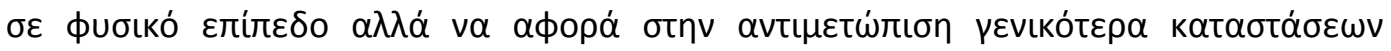

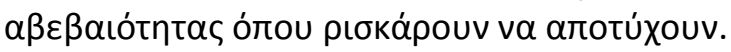

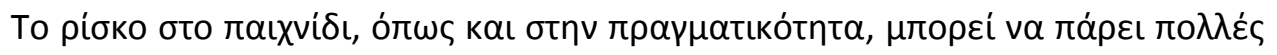

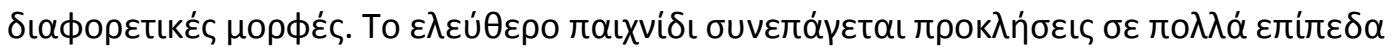

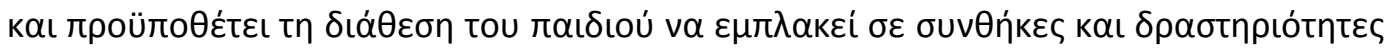

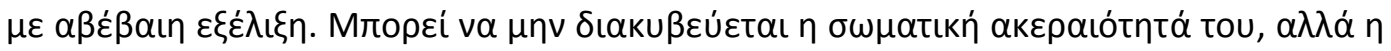

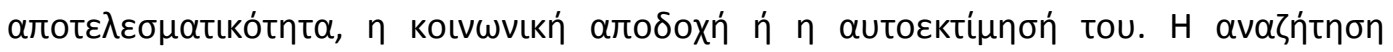

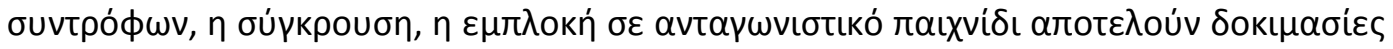

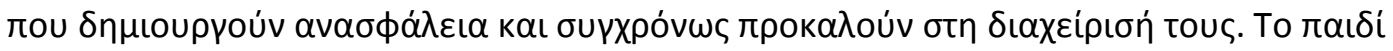

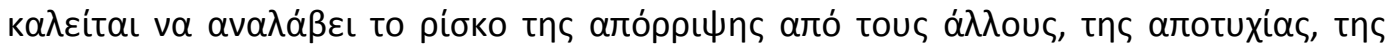

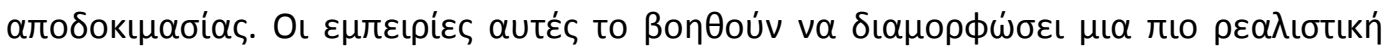

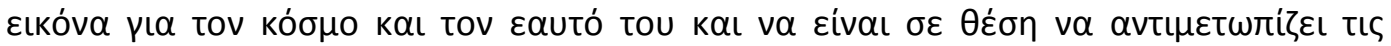

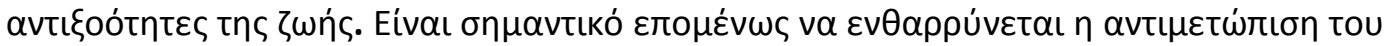

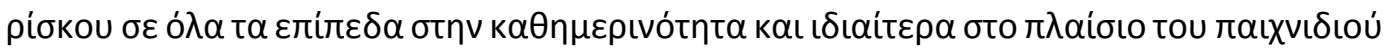

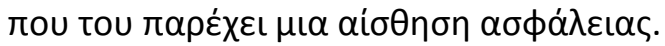

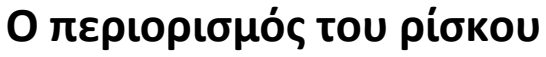

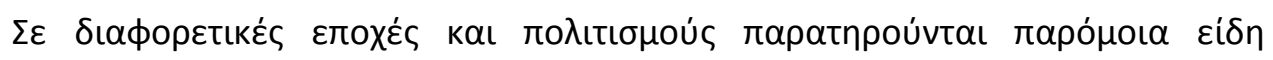

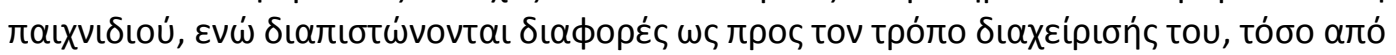

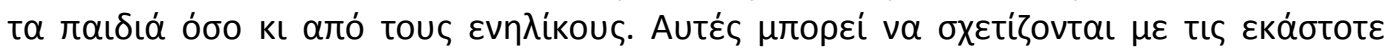

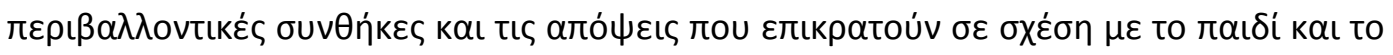

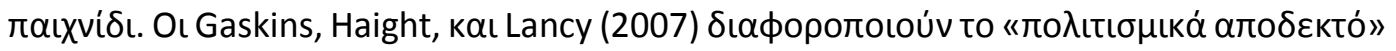

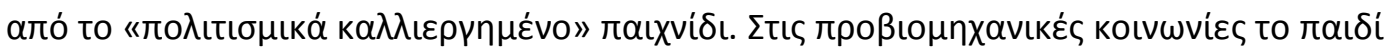

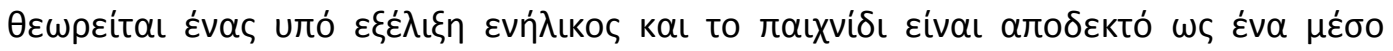

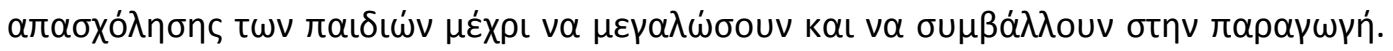

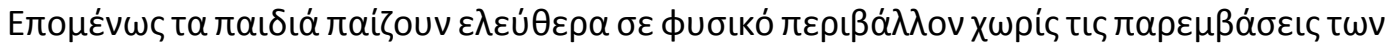

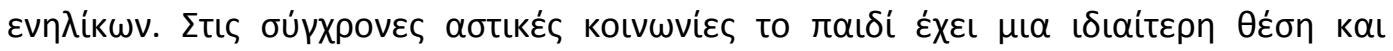

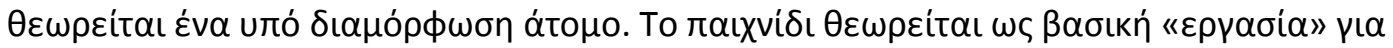

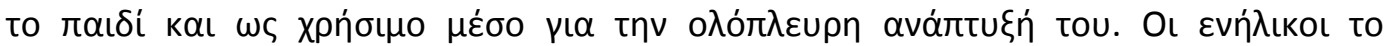

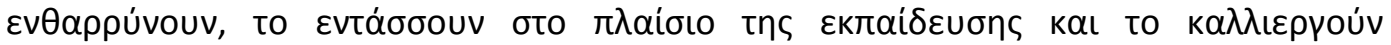

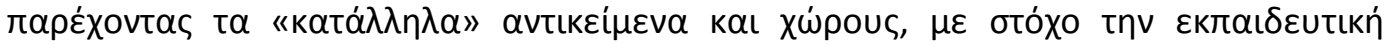

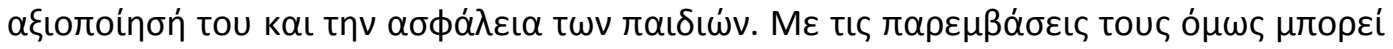

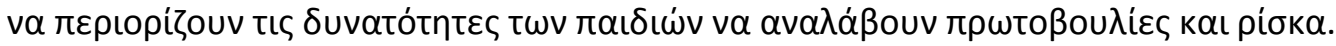




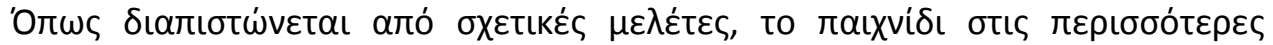

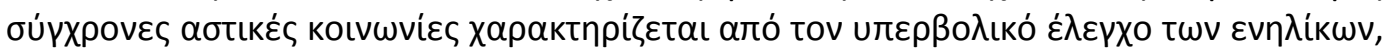

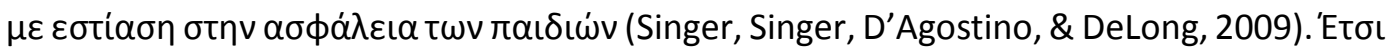

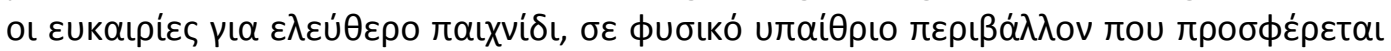

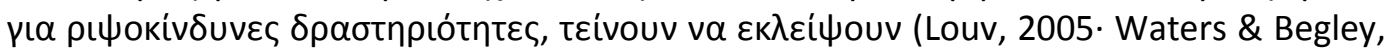

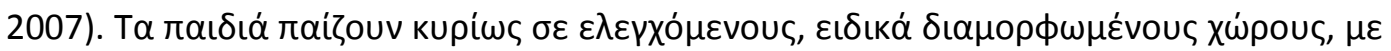

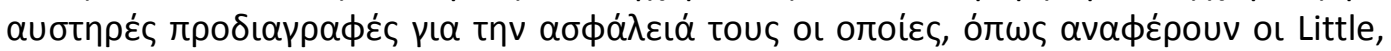

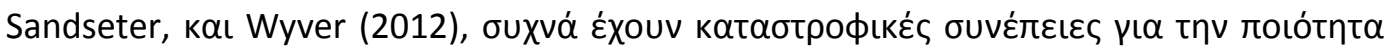

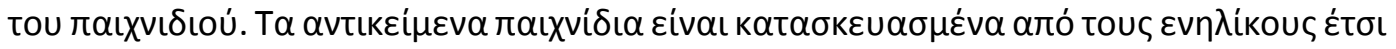

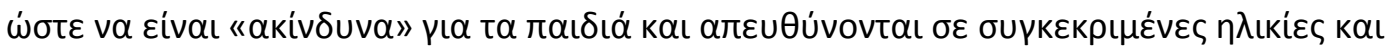

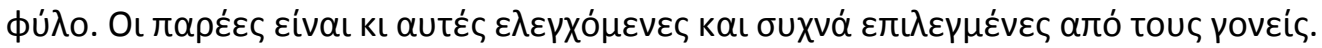

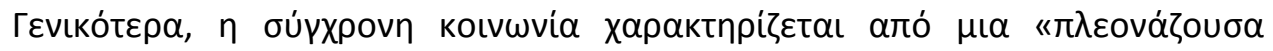

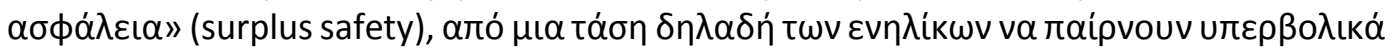

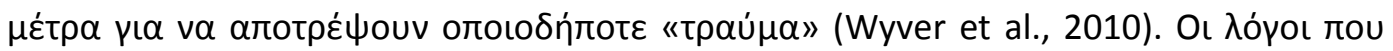

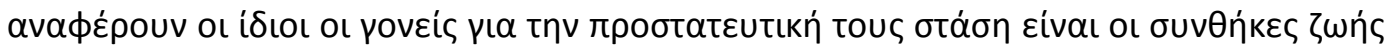

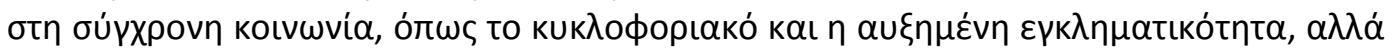

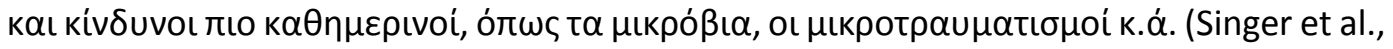

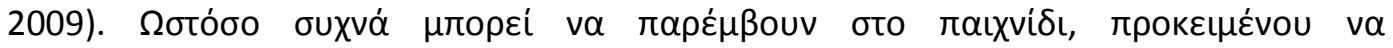

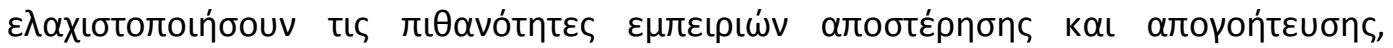

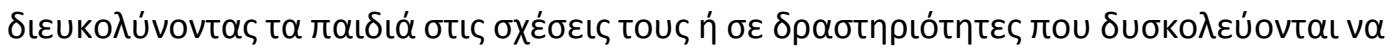

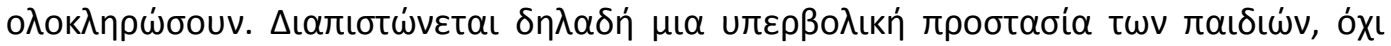

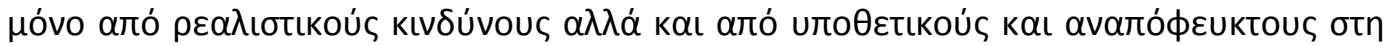

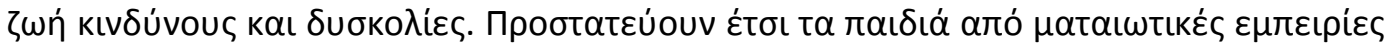

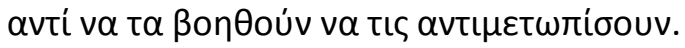

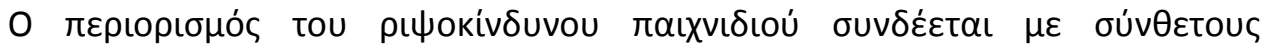

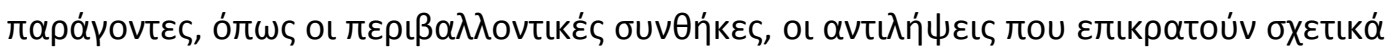

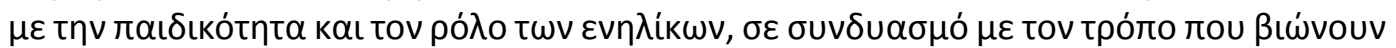

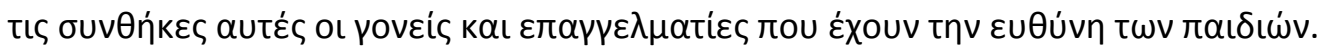

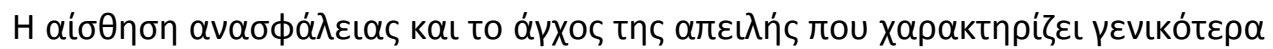

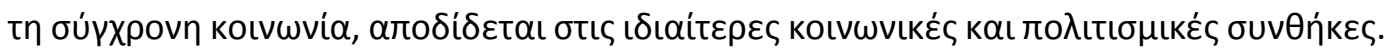

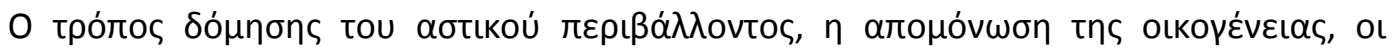

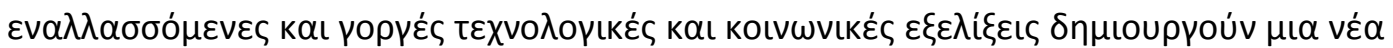

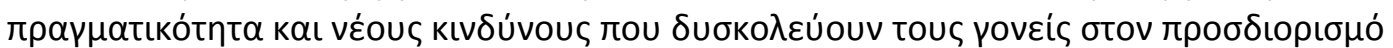

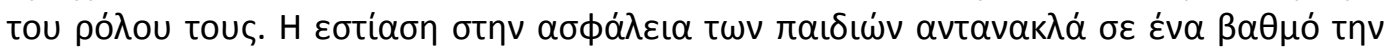

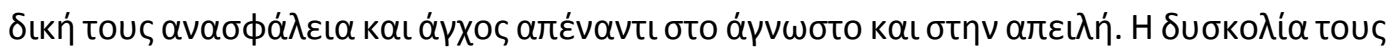

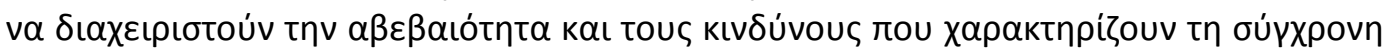

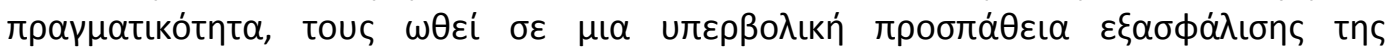

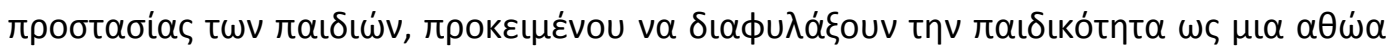

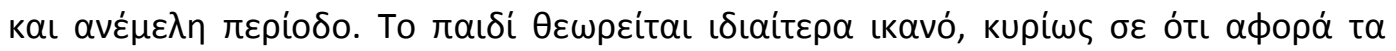

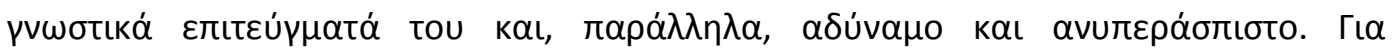

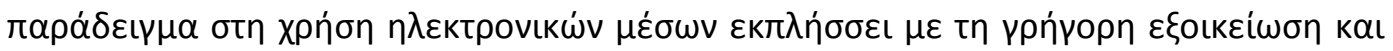

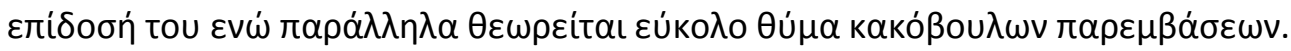

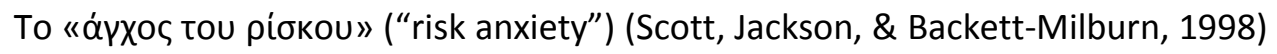

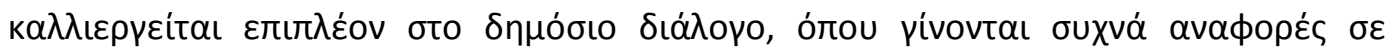

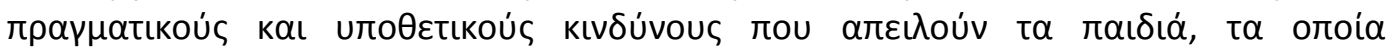

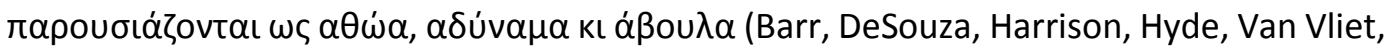




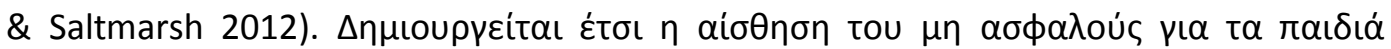

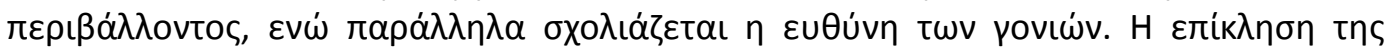

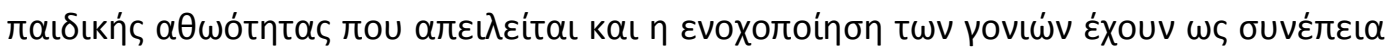

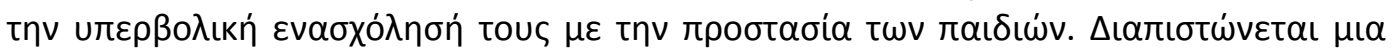

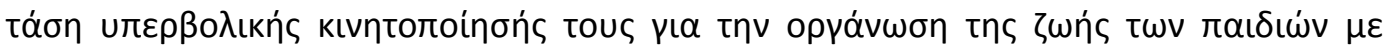

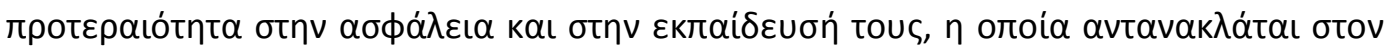

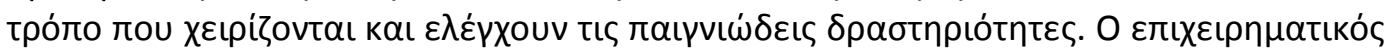

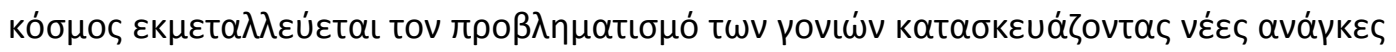

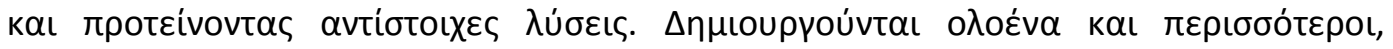

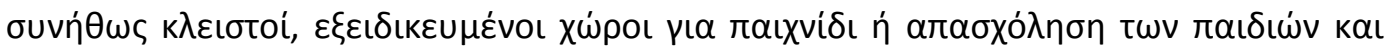

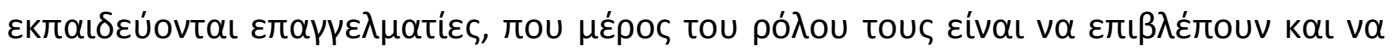

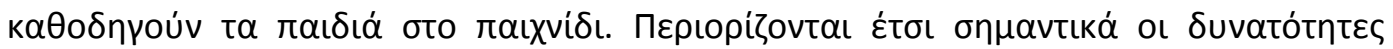

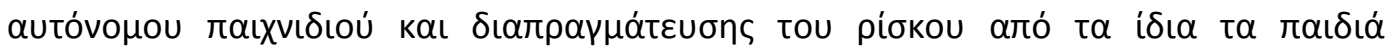

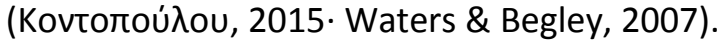

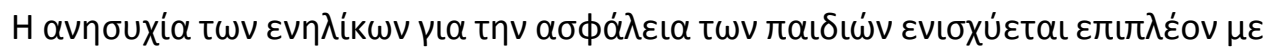

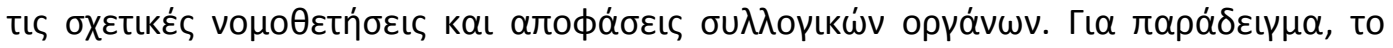

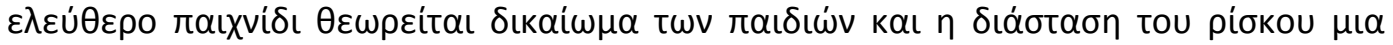

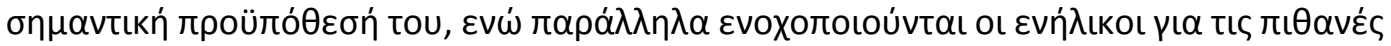

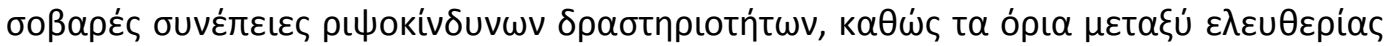

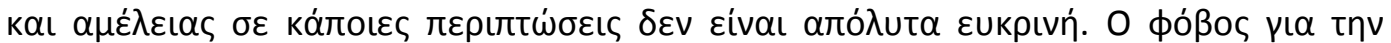

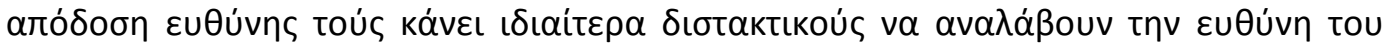

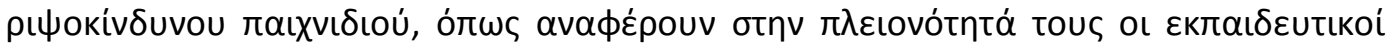

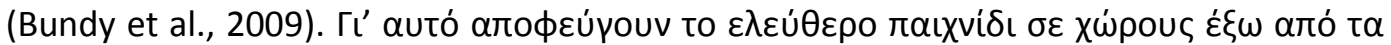

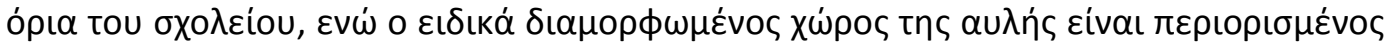

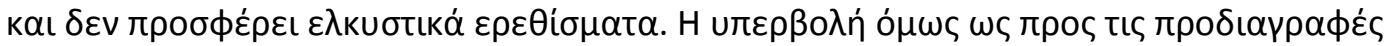

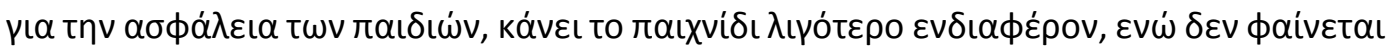

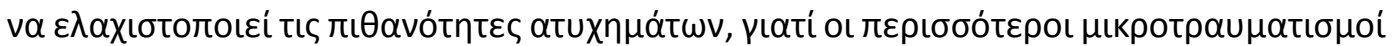

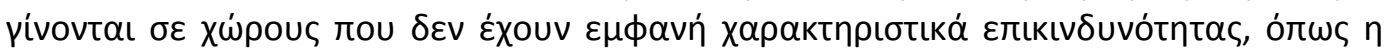

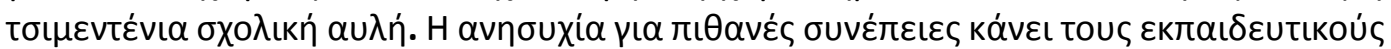

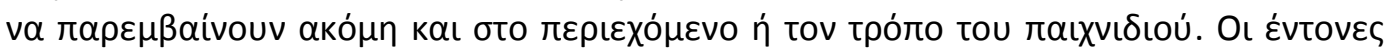

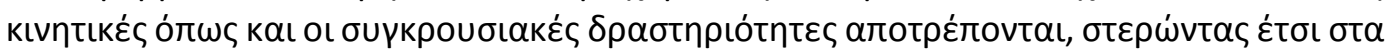

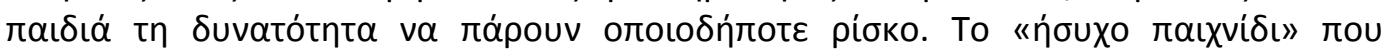

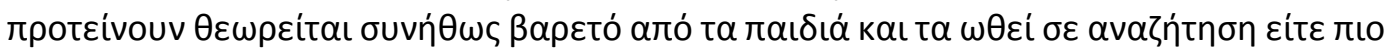

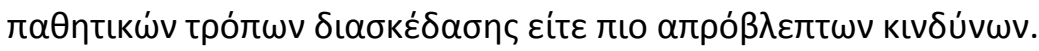

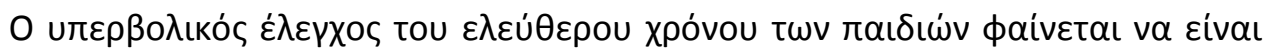

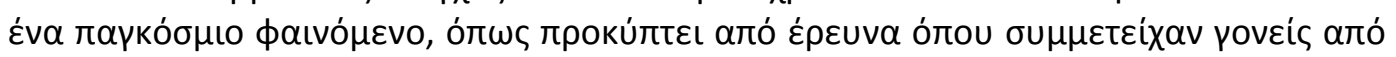

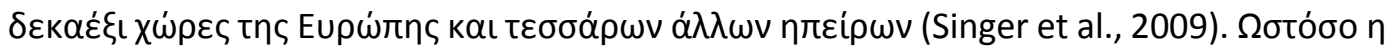

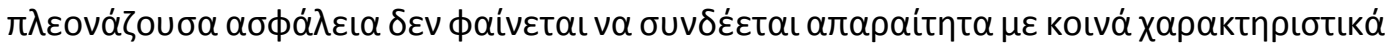

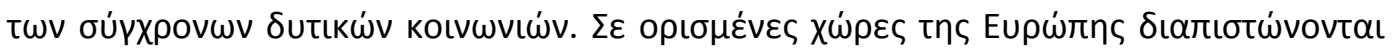

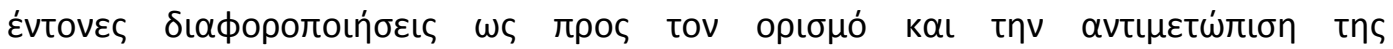

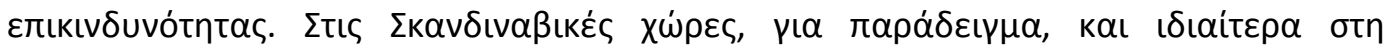

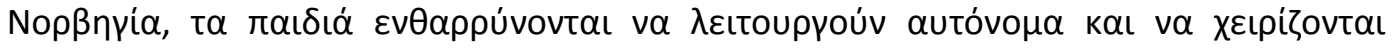

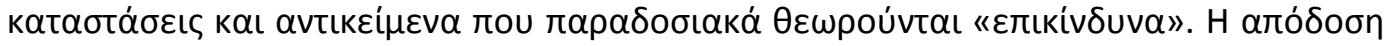

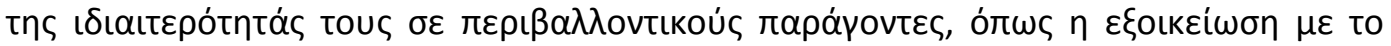

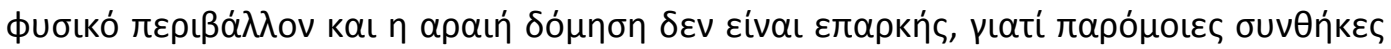

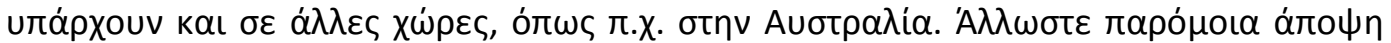

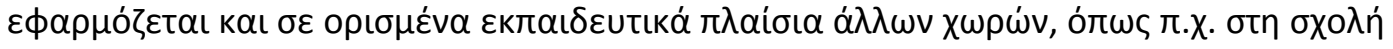




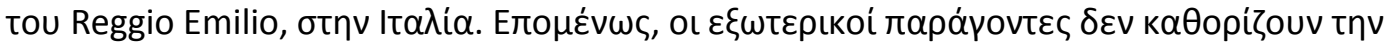

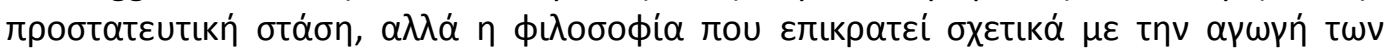
$\pi \alpha \iota \delta\llcorner\omega \dot{v}$.

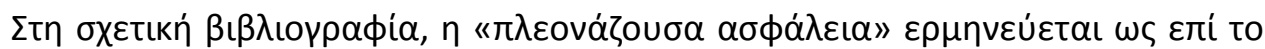

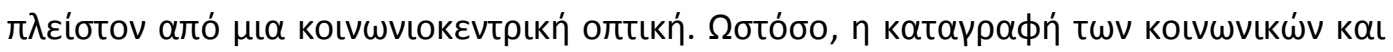

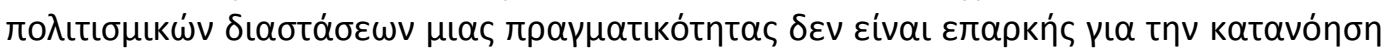

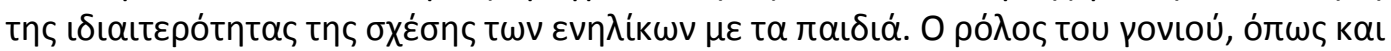

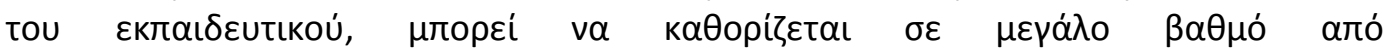

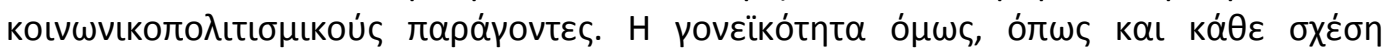

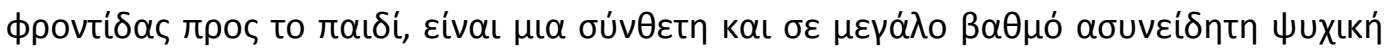

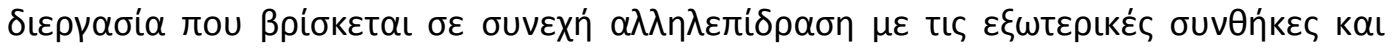

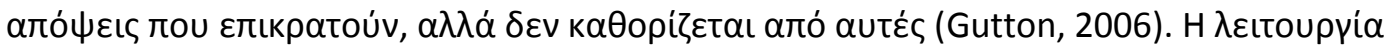

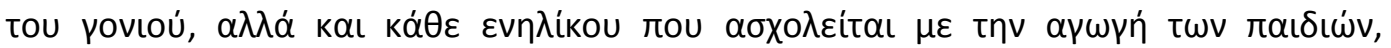

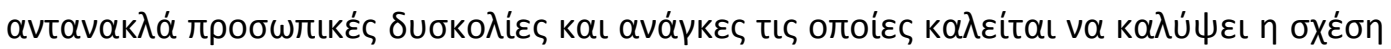

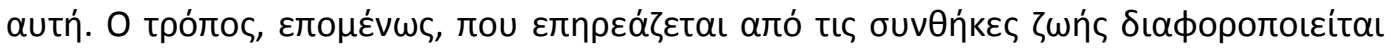
(Коvтотои́ภou, 2014).

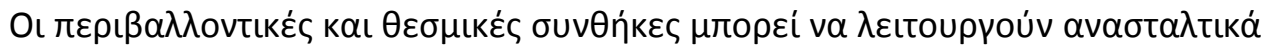

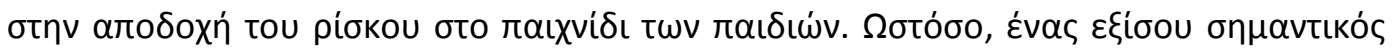

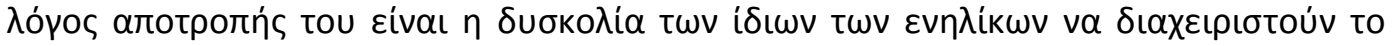

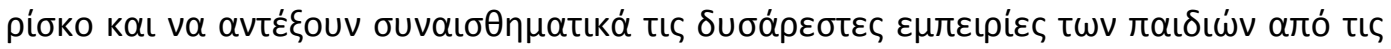

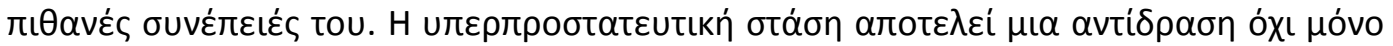

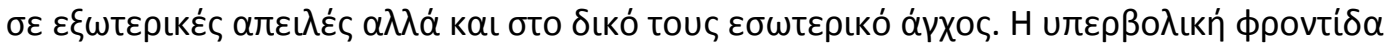

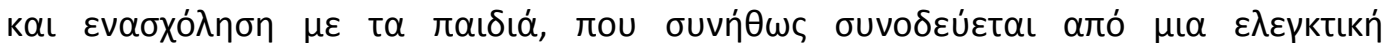

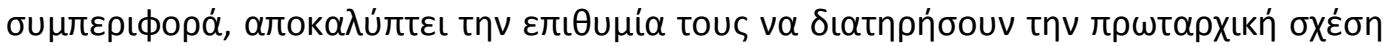

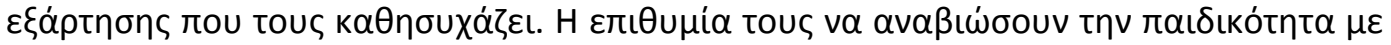

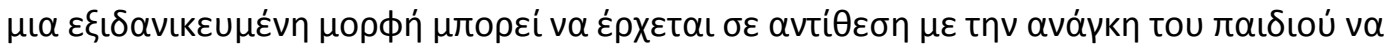

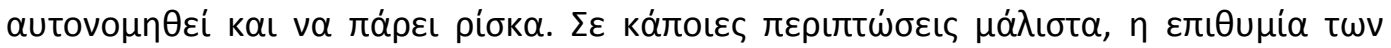

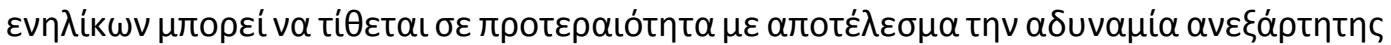

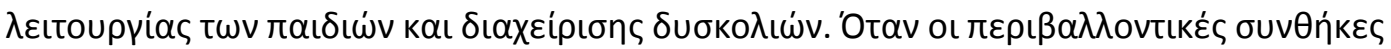

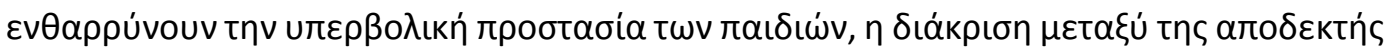

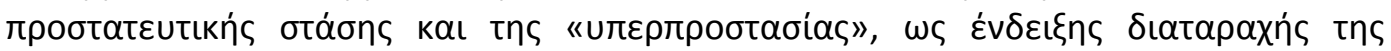

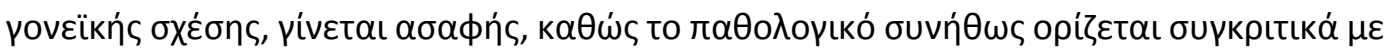

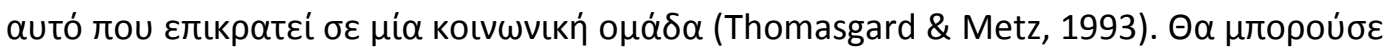

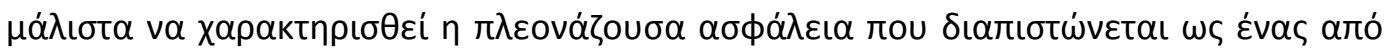

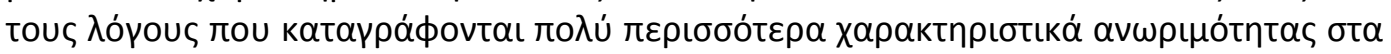

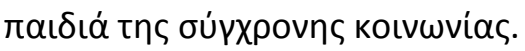

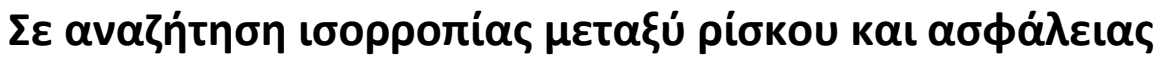

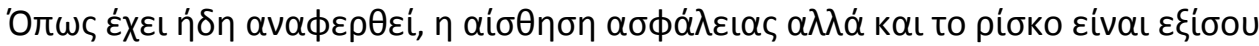

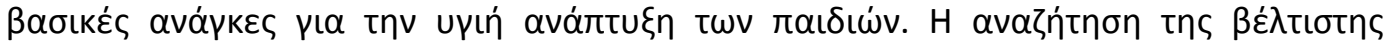

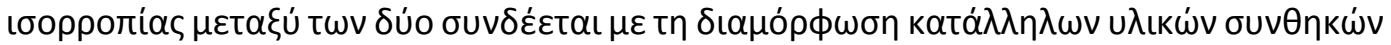

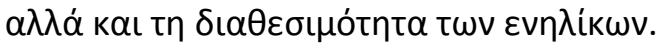

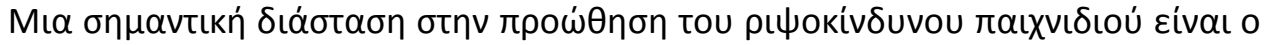

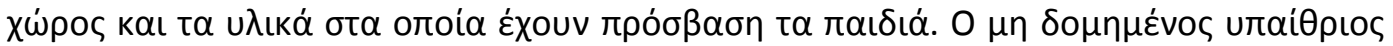

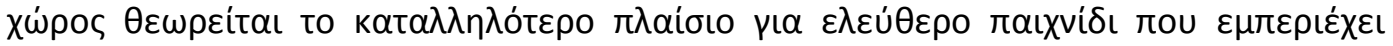




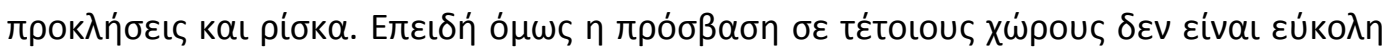

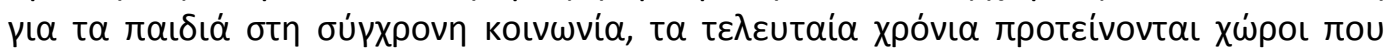

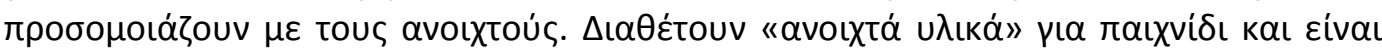

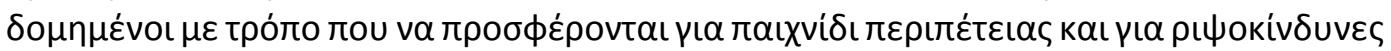

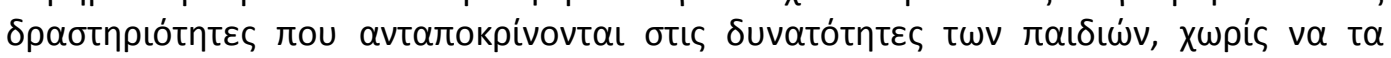

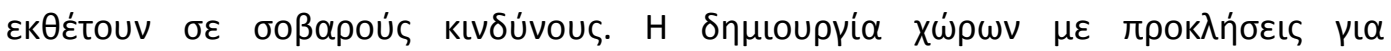

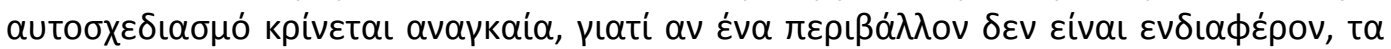

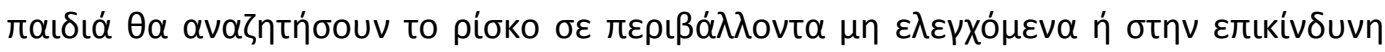

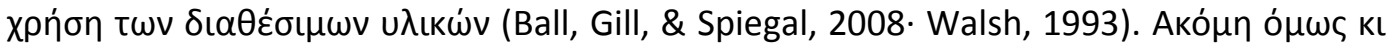

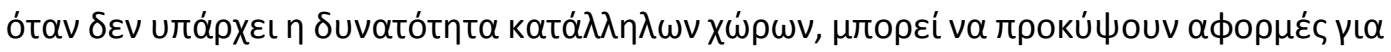

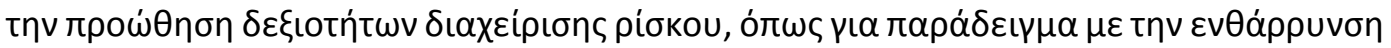

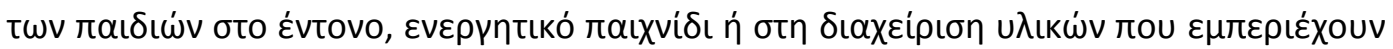

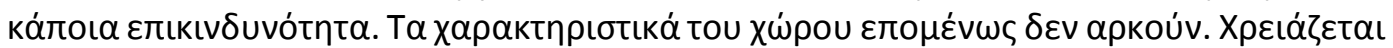

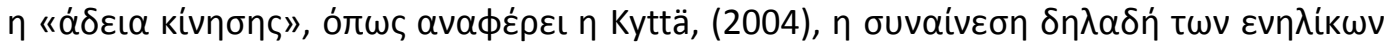

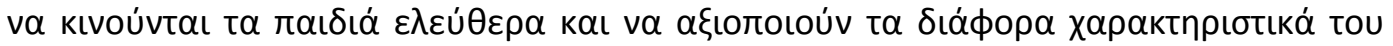

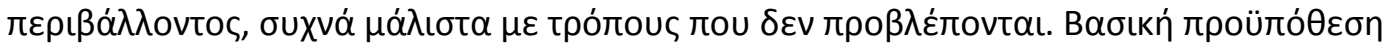

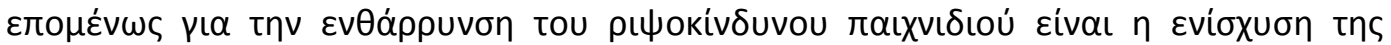

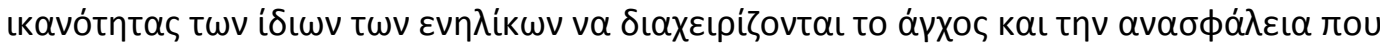

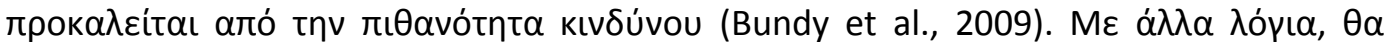

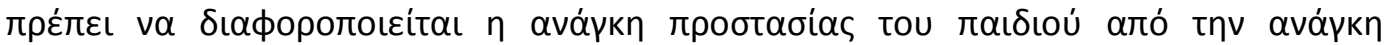

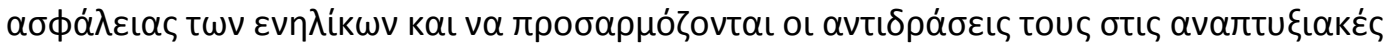

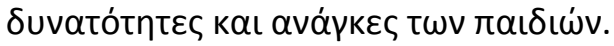

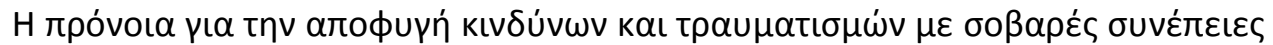

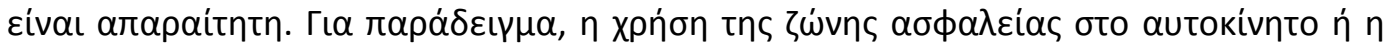

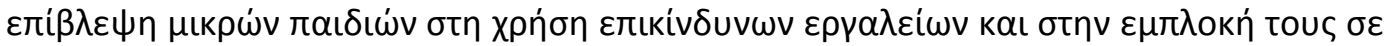

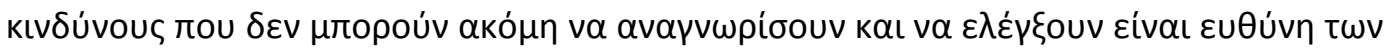

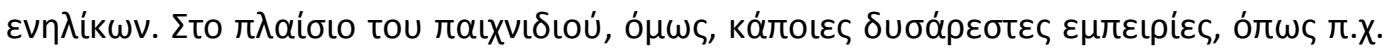

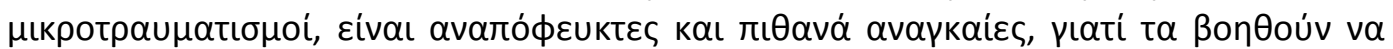

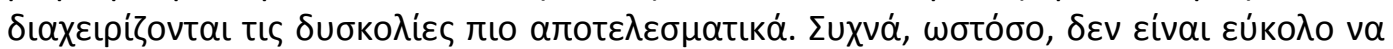

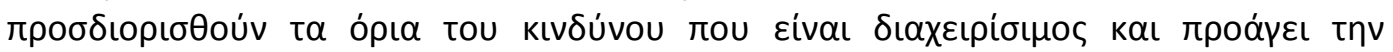

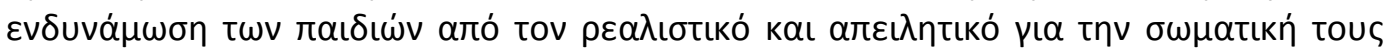

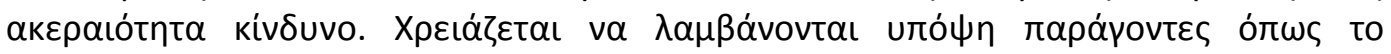

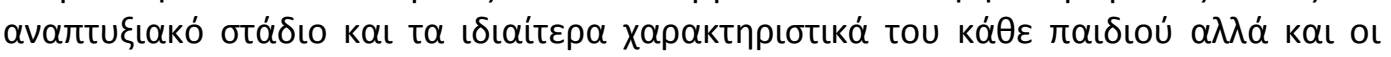

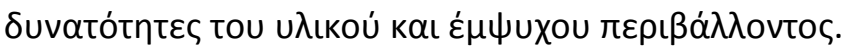

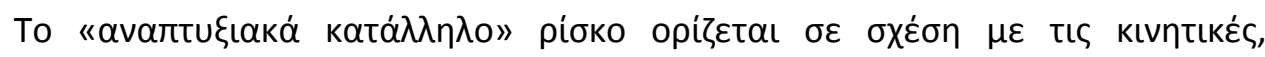

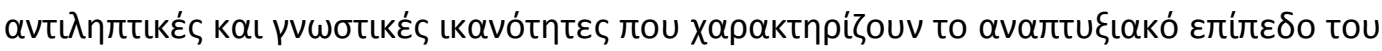

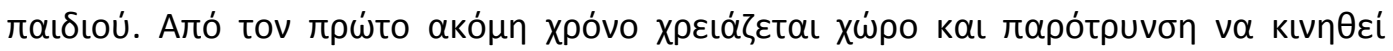

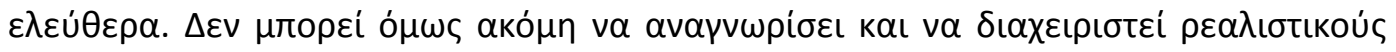

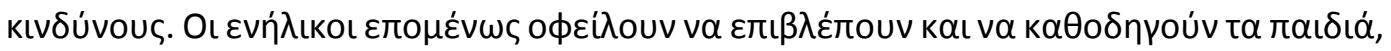

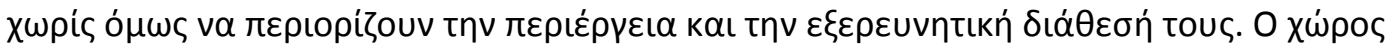

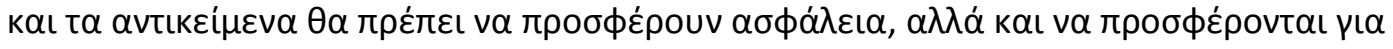

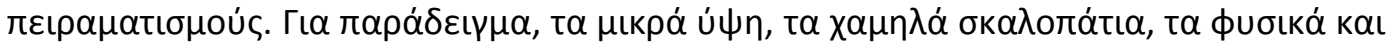

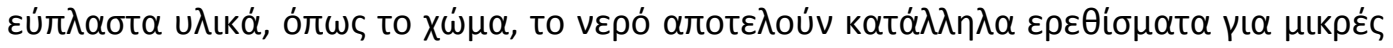

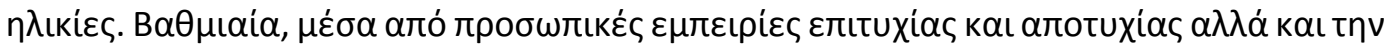

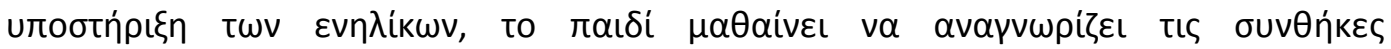

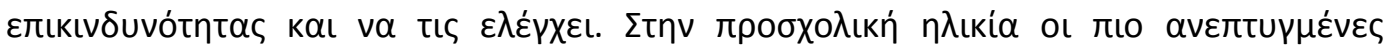

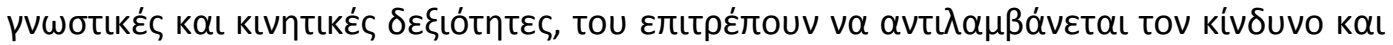




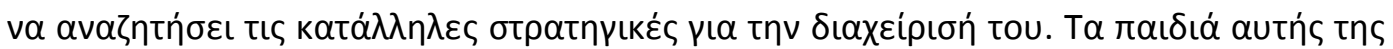

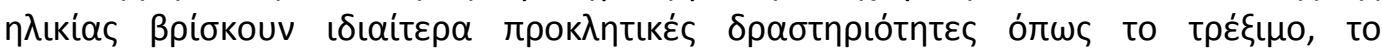

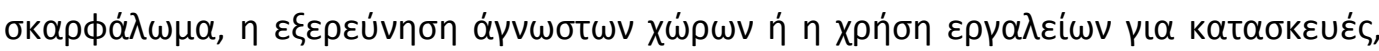

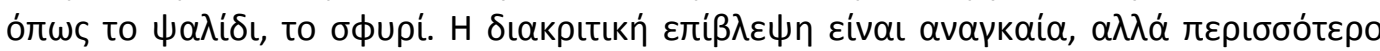

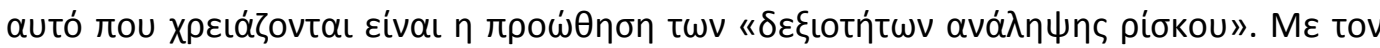

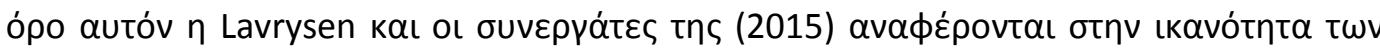

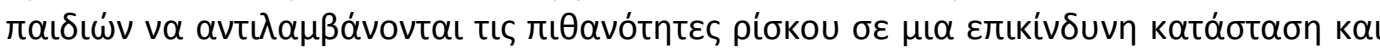

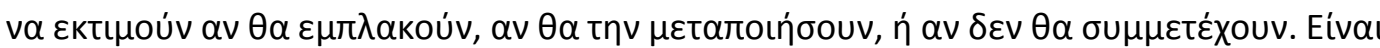

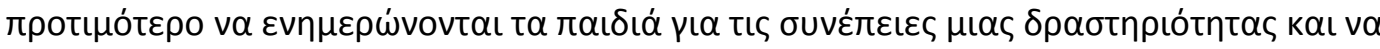

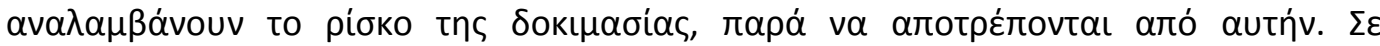

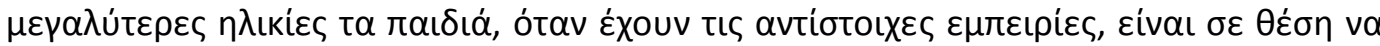

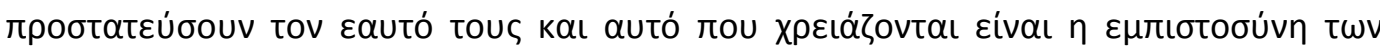
$\varepsilon \vee \eta \lambda i ́ \kappa \omega v$.

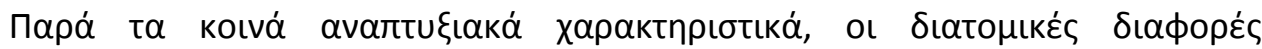

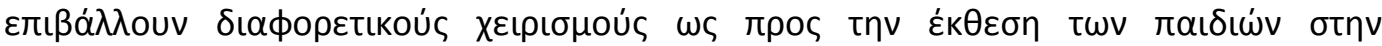

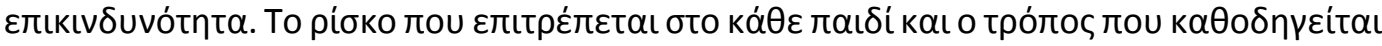

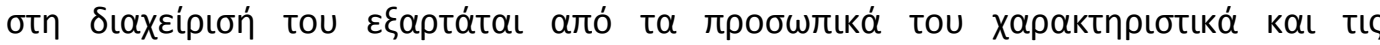

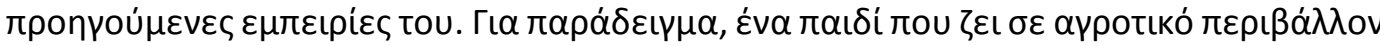

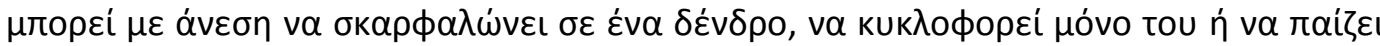

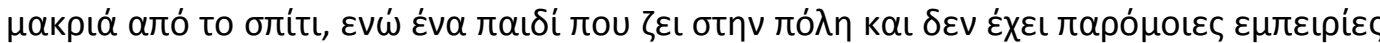

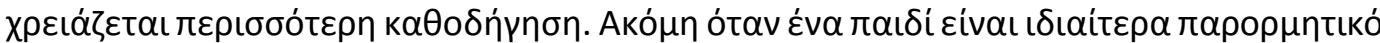

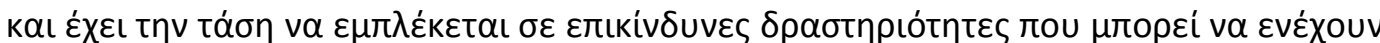

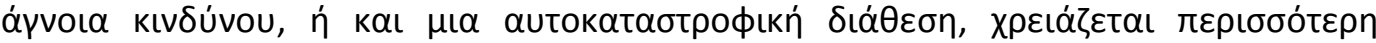

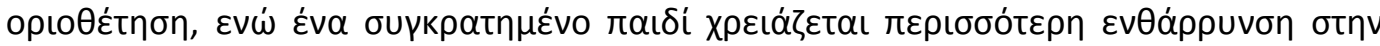

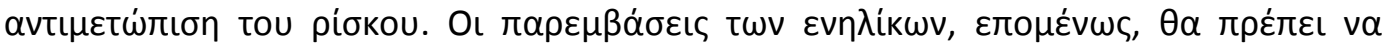

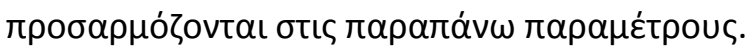

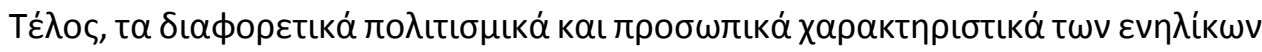

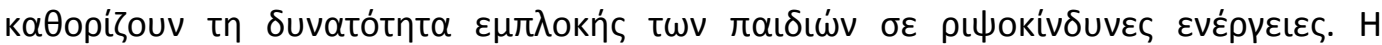

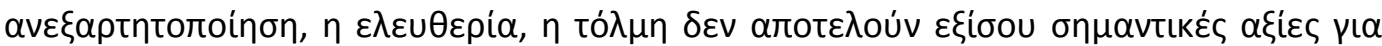

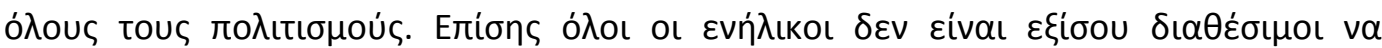

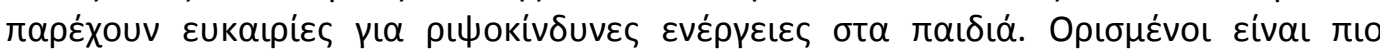

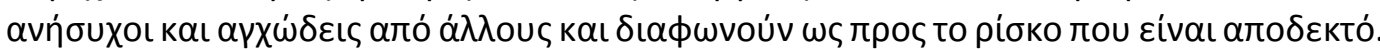

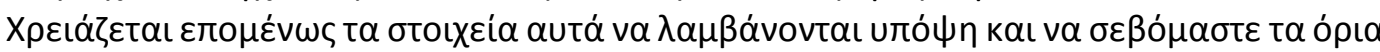

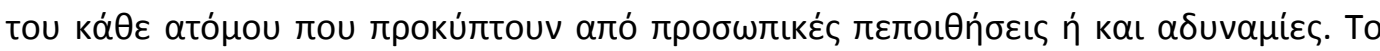

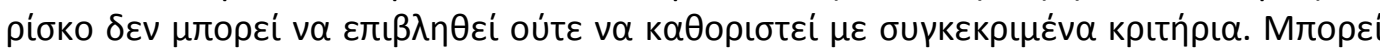

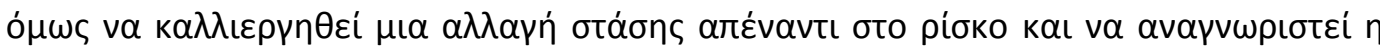

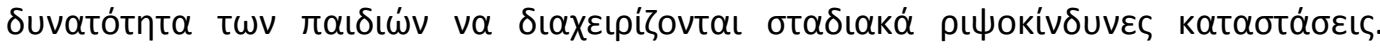

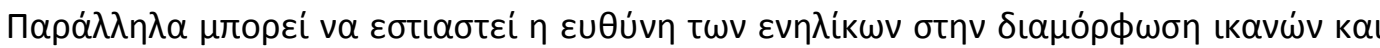

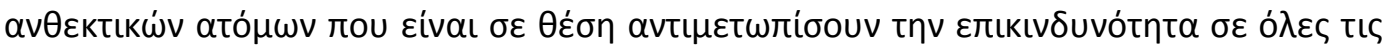

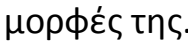

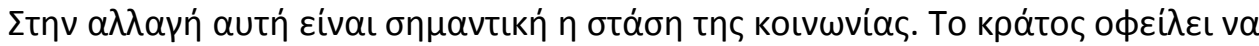

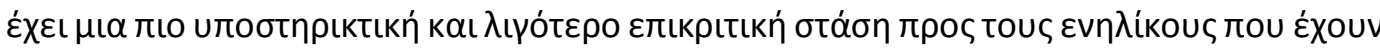

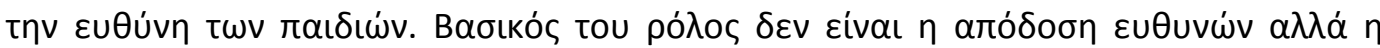

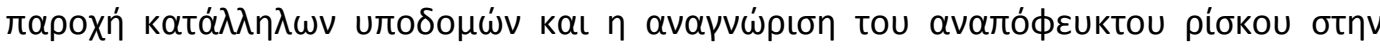

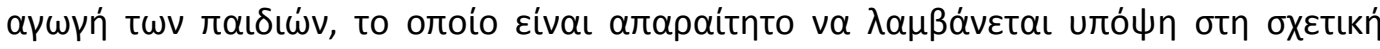

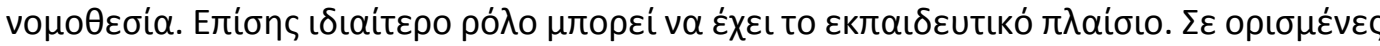




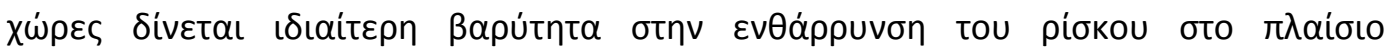

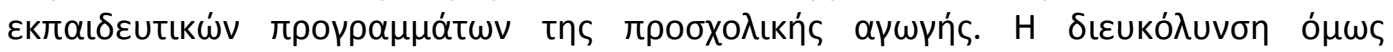

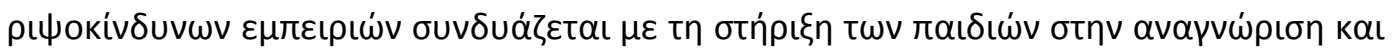

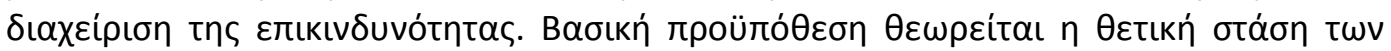

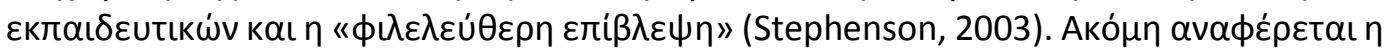

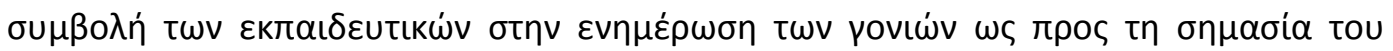

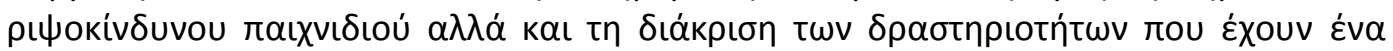
a

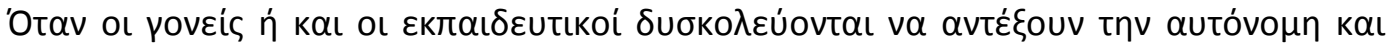

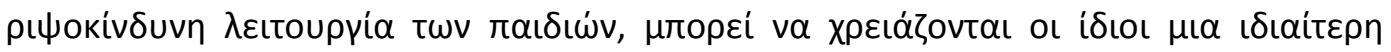

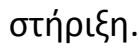

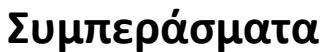

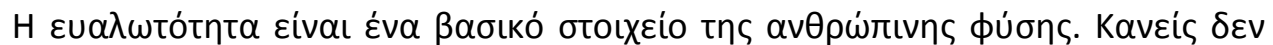

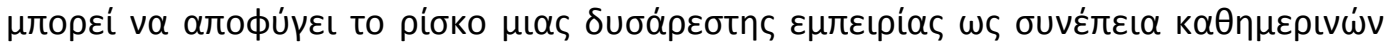

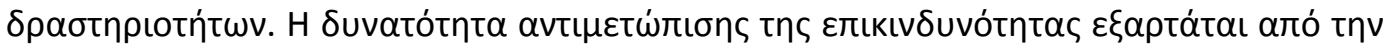

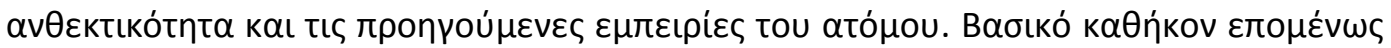

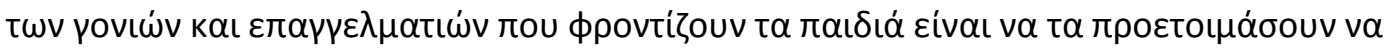

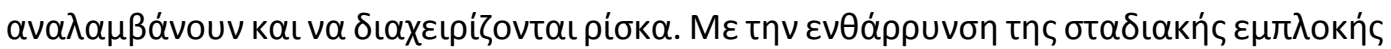

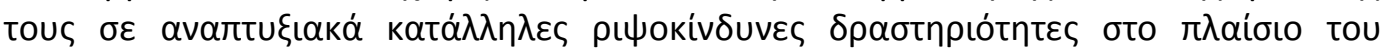

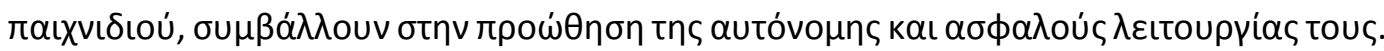

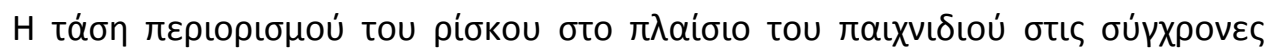

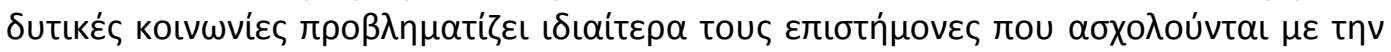

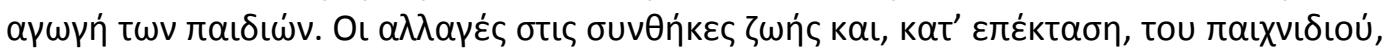

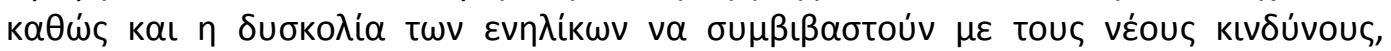

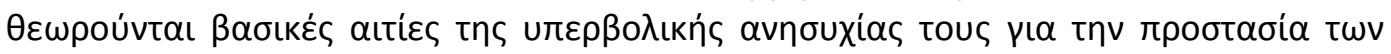

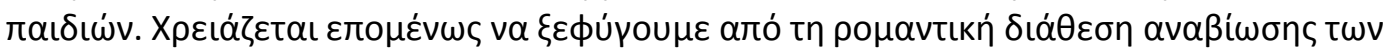

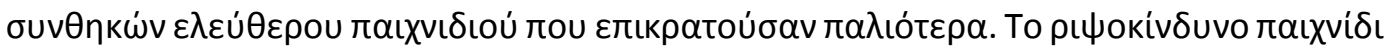

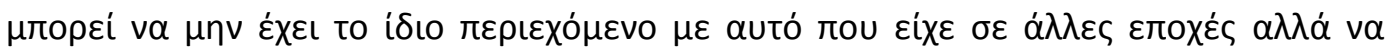

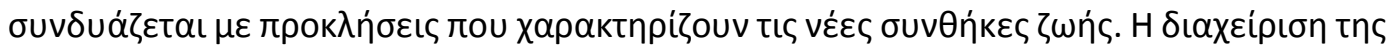

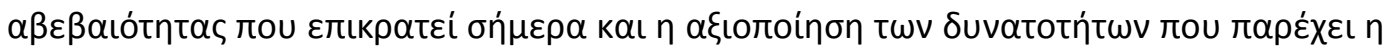

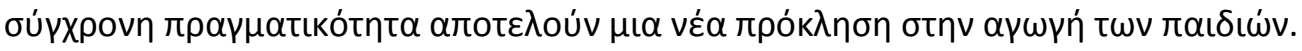

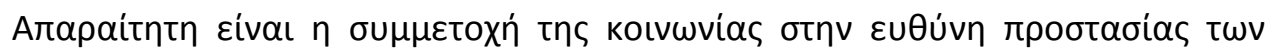

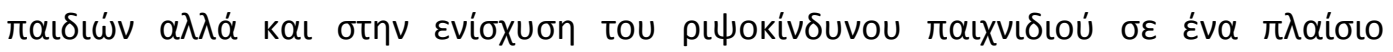

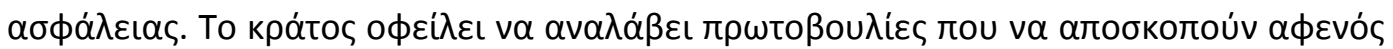

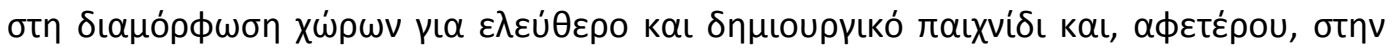

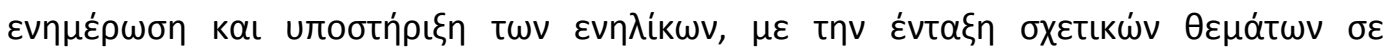

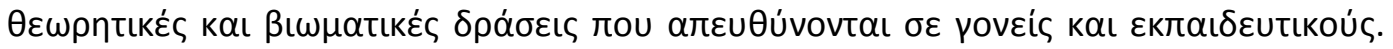

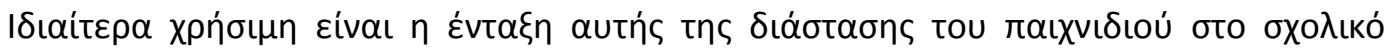

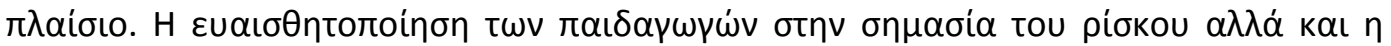

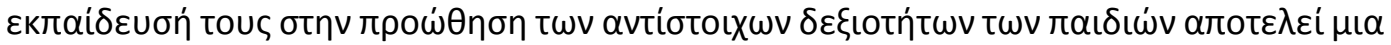

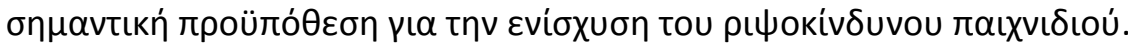

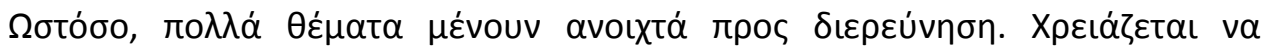

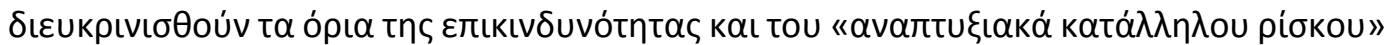

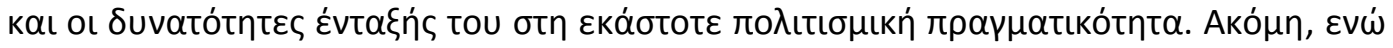




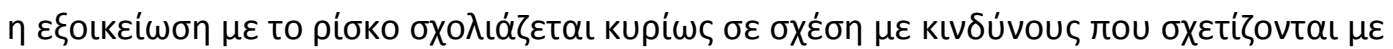

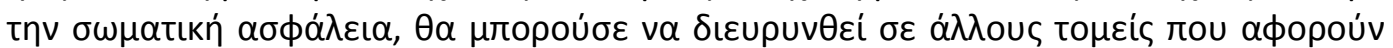

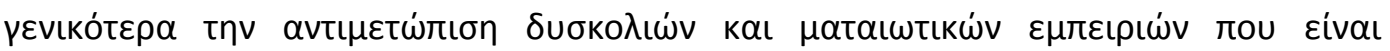

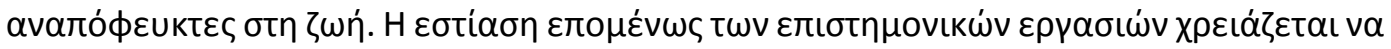

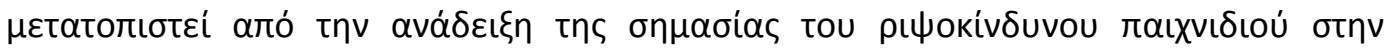

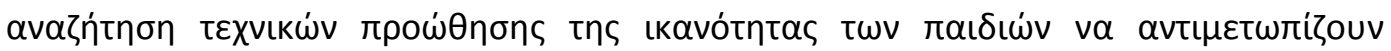

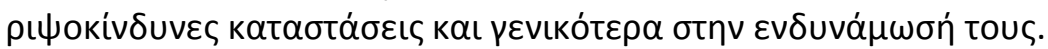

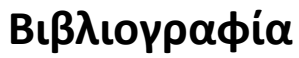

Ball, D., Gill, T., \& Spiegal, B. (2008). Managing risk in play provision: Implementation guide. Nottingham: DCSF Publications.

Barr, J., De Souza, S., Harrison, C., Hyde, B., van Vliet, H., \& Saltmarsh, S. (2012). Parenting the 'Millennium Child': choice, responsibility and playing it safe in uncertain times. Global Studies of Childhood, 2(4), 302-318. http://dx.doi.org/10.2304/gsch.2012.2.4.302

Bundy, A. C., Luckett, T., Tranter, P. J., Naughton, G. A., Wyver, S. R., Ragen, J., \& Spies, G. (2009). The risk is that there is 'no risk': a simple, innovative intervention to increase children's activity levels. International Journal of Early Years Education, 17(1), 33-45.

Carr, M. (2001). Assessment in early childhood settings: Learning stories. London: Paul Chapman.

Costa, A. L. (1991). The search for intelligent life. In A. L. Costa (Ed.), Developing minds: A resource book for teaching thinking. Revised Edition. Vol. 1. (pp. 100-106). Alexandria, USA: Association for Supervision and Curriculum Development. Retrieved from http://files.eric.ed.gov/fulltext/ED332166.pdf

Gaskins, S., Haight, W., \& Lancy, D. F. (2007). The cultural construction of play. In A. Göncü \& S. Gaskins (Eds.), Play and development: Evolutionary, sociocultural and functional perspectives (pp. 179-202). Mahwah, NJ: Lawrence Erlbaum.

Gutton, P. (2006). Parentalité. Adolescsence, 1(55), 9-32.

Howard, R. C. (2011). The quest for excitement: A missing link between personality disorder and violence? Journal of Forensic Psychiatry \& Psychology, 22(5), 692-705.

Jarvis, P. (2007). Dangerous activities within an invisible playground: A study of emergent male football play and teachers' perspectives of outdoor play in the early years of primary school. International Journal of Early Years Education, 15(3), 245-259.

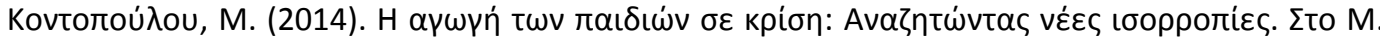

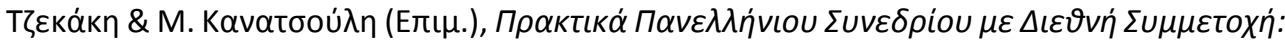

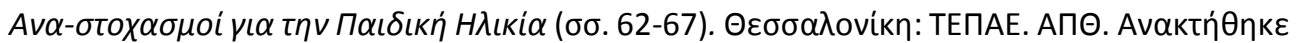
aró http://www.nured.auth.gr/congress2014

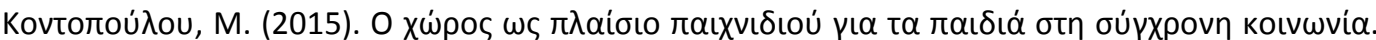

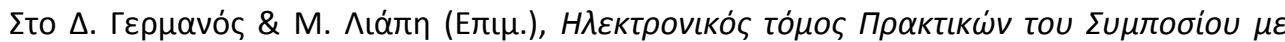

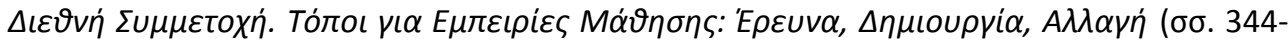

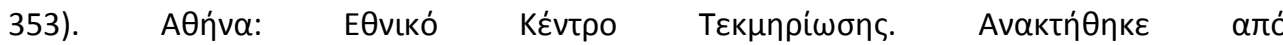
http://epublishing.ekt.gr/el/12239

Kyttä, M. (2004). The extent of children's independent mobility and the number of actualized affordances as criteria for child-friendly environments. Journal of Environmental Psychology, 24, 179-198. 


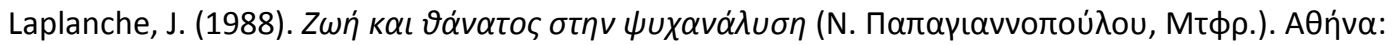

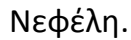

Lavrysen, A., Bertrands, E., Leyssen, L., Smets, L., Vanderspikken, A., \& De Graef, P. (2015). Risky play at school. Facilitating risk perception and competence in young children. European Early Childhood Education Research Journal, 25, 1-17.

Le Heuzey, M. F. (2009). Jeux dangereux: Quand l'enfant prend des risques. Paris: Odile Jacob.

Little, H., Sandseter, E. B. H., \& Wyver, S. (2012). Early Childhood Teachers' Beliefs about Children's Risky Play in Australia and Norway. Contemporary Issues in Early Childhood, 13(4), 300-316.

Louv, R. (2005). Last child in the woods: Saving our children from nature deficit disorder. Chapel Hill, NC: Algonquin Books.

Olivier, S. (2006). Moral dilemmas of participating in dangerous leisure activities. Leisure studies, 25(1), 95-109.

Pelegrini, A., \& Smith, P. K. (1998). Physical activity play: the nature and function of a neglected aspect of play. Child Development, 69, 557-598.

Sandseter, E. B. H. (2010). 'it tickles in my tummy!' understanding children's risk-taking in play through reversal theory. Journal of Early Childhood Research, 8(1), 67-88.

Sandseter, E. B. H. (2012). Restrictive safety or unsafe freedom? Norwegian ECEC practitioners' perceptions and practices concerning children's play risky play. Child Care in Practice, 18(1), 83-101.

Sandseter, E. B. H. (2014). Early childhood education and care practitioners' perceptions of children's risky play; examining the influence of personality and gender. Early Child Development And Care, 184(3), 434-449. http://dx.doi.org/10.1080/03004430.2013.794797

Sandseter, E. B. H., \& Kennair, L. E. O. (2011). Children's risky play from an evolutionary perspective: The anti-phobic effects of thrilling experiences. Evolutionary Psychology, 9(2), 257-284.

Scott, S., Jackson, S., \& Backett-Milburn, K. (1998). Swings and roundabouts: risk anxiety and the everyday worlds of children. Sociology, 32(4), 689-705.

Singer, D. G., Singer, J. L., D’Agostino, H., \& DeLong, R. (2009). Children's pastimes and play in sixteen nations: is free-play declining? American Journal of Play, 1(3), 283-312.

Smith, S. J. (1998). Risk and our pedagogical relation to children: On playground and beyond. New York: Guilford.

Stephenson, A. (2003). Physical risk taking: Dangerous or endangered? Early Years, 23(1), 35-43.

Stine, S. (1997). Landscapes for learning: creating outdoor environments for children and youth. New York: Wiley.

Thomasgard, M., \& Metz, W. P (1993). Parental overprotection revisited. Child Psychiatry and Human Development, 24(2), 67-80. Retrieved from http://gsc.sagepub.com/content/2/4/302.full.pdf+html

Walsh, P. (1993). Fixed equipment-a time for change. Australian Journal of Early Childhood, 18(2), 23-29.

Waters, J., \& Begley, S. (2007). Supporting the development of risk taking behaviors in the early years: An exploratory study. Education 3-13, 35(4), 365-377. 
Whitebread, D., Basilio, M., Kuvalja, M., \& Verma, M. (2012). The importance of play: A report on the value of children's play with a series of policy recommendations. Brussels, Belgium: Toys Industries for Europe. Retrieved from http://www.importanceofplay.eu/IMG/pdf/dr david whitebread the importance of play.pdf

Wyver, S., Tranter, P., Naughton, G., Little, H., Sandseter, E. B. H., \& Bundy, A. (2010). Ten ways to restrict children's freedom to play: the problem of surplus safety. Contemporary Issues in Early Childhood, 11(3), 263-277. 